\title{
High-performance carbon black/molybdenum disulfide nanohybrid sensor for cocoa catechins determination using an extraction-free approach
}

Flavio Della Pelle $e^{a}$, Daniel Rojass ${ }^{\mathrm{a}, \mathrm{b}}$, Annalisa Scroccarello ${ }^{\mathrm{a}}$, Michele Del Carlo ${ }^{\mathrm{a}}$, Giovanni Ferraro ${ }^{\mathrm{c}}$, Carla Di Mattia Maria Martuscellia ${ }^{\mathrm{a}}$, Alberto Escarpa ${ }^{\mathrm{b}, \mathrm{d}^{*}}$, Dario Compagnone $\mathrm{a}^{\mathrm{a}^{* *}}$

${ }^{a}$ Faculty of Bioscience and Technology for Food, Agriculture and Environment University of Teramo 64023, Teramo (Italy). Fax:(+39) 0861-266942, e-mail: **dcompagnone@ unite.it

${ }^{\mathrm{b}}$ Department of Analytical Chemistry, Physical Chemistry and Chemical Engineering, Faculty of Sciences University of Alcalá, E-28871 Alcalá de Henares, Madrid, Spain. Fax: (+34) 918854971, e-mail: *alberto.escarpa@uah.es

'Department of Chemistry "Ugo Schiff” and CSGI, University of Florence, Via della Lastruccia 3-Sesto Fiorentino, I50019, Florence, Italy.

${ }^{\mathrm{d}}$ Chemical Research Institute “Andres M. del Rio”, University of Alcalá, E-28871, Madrid, Spain

\section{Abstract}

In this work, for the first time, the ability to detect catechins on an effective and regenerable carbon black/molybdenum disulfide nanohybrid screen-printed electrode $\left(\mathrm{SPE}-\mathrm{CB} / \mathrm{MoS}_{2}\right)$ is demonstrated. The proposed SPE-CB/MoS 2 merges the ability of $\mathrm{CB}$ to improve the electrochemical response with the proprieties of $\mathrm{MoS}_{2}$ to totally prevent catechins irreversible polymerization and adsorption onto the electrode surface occurring at both bare and CB-modified SPEs. The $\mathrm{MoS}_{2}$ antifouling ability has been demonstrated using both flavanols standards and complex samples thus proved to be an unexpected and useful characteristic. The SPE-CB/MoS 2 allowed an improvement of sensitivity (LOD $\leq 0.17 \mu \mathrm{mol} \mathrm{L}^{-1}$ ) of 100-folds compared to the bare SPE electrode, showing linear range between 0.12 and $25 \mu \mathrm{mol} \mathrm{L} \mathrm{L}^{-1}$ with good determination coefficients $\left(R^{2} \geq 0.998\right)$. Cocoa powder samples have been analyzed employing the developed hybrid sensor and compared with classical methods for polyphenols evaluation as Folin- Ciocalteu (F.C.), ABTS and an AuNPs based assay. The SPE-CB/MoS 2 allow to obtain repeatable and reproducible results which also were significantly correlated with classical methods ( $\mathrm{r}=0.95-0.97)$. Noteworthy, after the measurements of 59 cocoa samples the electrode was still active (recovery signal $99 \%$ ). Using the extraction-free approach with the SPE-CB/MoS 2 sensor the whole analysis of cocoa powder catechins required only 15 minutes.

Keywords: hybrid sensor; catechins analysis; carbon black; molybdenum disulfide; transition metal dichalcogenide; cocoa samples.

\section{Introduction}

Recent trends in food marketing suggest that cocoa and cocoa-products present multiple-benefits which are becoming subject of increased scientific research, mainly because of their phytochemical composition [1,2]. In fact, the high polyphenols content of cocoa has generated a renewed interest for nutritional and health viewpoints [3], and thanks to this properties, the global demand for cocoa is increasing [4]. In the cocoa-based products market, the cocoa content results to be an added value, influencing price and the organoleptic properties [5]. Cocoa is rich in flavanols (catechins) and proanthocyanidins, strong antioxidants, which have been associated with several potential health benefits $[1,3]$. Moreover, they also affect the cocoa fat resistance towards oxidation, during the production process and conservation $[3,6-8]$. In fact, the final content of antioxidant compounds in cocoa-based products is a function of several variables related to the raw material and others related to processing and formulation [3,6-8].

Cocoa catechins contents and their antioxidant capacity evaluation are still a challenging issue, in particular for the complexity of the matrix. Indeed, the extraction step is necessary to remove the possible interfering compounds, separate the polyphenols from the lipids, but can lead to low recovery, resulting in consumption of solvents and time [9]. The 
cocoa polyphenolic extracts classically are analyzed with spectrophotometric assays or chromatographic methods [2,3,10-14]; the latter can provide identification of the constituents and amount, but do not returns direct information on the antioxidant capacity of polyphenols [15]. Classical spectrophotometric methods are simple but can present some disadvantages as the use of expensive reagents, not environmentally friendly (i.e. radical compounds, metals, etc.), undefined reaction time, long sample pretreatment, and low precision, selectivity, and sensitivity [16]. Several other methods have been proposed for polyphenols cocoa analysis, as e.g. capillary electrophoresis [17], NIR [18] and FT-IR spectroscopy [19], and only few methods don't require sample preparation and extraction [9].

Electrochemical sensors represent a valuable alternative approach to the detection of polyphenols and antioxidant capacity $[15,16]$ and different electrochemical approaches for the direct sensing using several electrodes/transducers types and configurations have been proposed [15,16,20-22]. However, direct electrochemical oxidation of polyphenols onto classical unmodified carbon-based materials is affected by the typical drawback of fouling $[15,23]$. This phenomenon is particularly relevant for flavanols (catechins) structures [24]. In fact, the redox behavior of catechins onto carbon electrodes, despite being initially apparently reversible, results in an electrode surface irreversible passivation [25]. The passivation is caused by adsorption and polymerization of oxidation products onto the working electrode surface $[15,23]$. This drawback causes loss of sensitivity and reproducibility and drives versus the disposable use of the electrodes. Nanomaterials (NMs) have attracted attention since they can reduce the passivation of the electrode area, potentially improving also sensitivity and selectivity $[15,26-30]$. Different NMs have been used for catechins direct sensing purposes, as nanotubes [31], graphene [32,33], etc. [24,34-38]. However, the catechins by-products of oxidation tendency to interact with the electrode surface is strong and this does not allow reproducible and accurate analysis. In fact, very often the electrochemical behavior of the catechins is studied as proof of concept, without a deep study of both passivation and exploitability with complex samples [15]. This drawback and the need of an extraction step have limited the use of electrochemical sensors in this field, for example, as very few application of sensors on cocoa samples are reported in literature [35,38].

In the last years, carbon black (CB) has been widely employed for building electrochemical platforms. Its particular features stimulated intense research efforts; in fact, CB nanospheres possess high surface area, high electrical conductivity, low cost, and high dispersibility and can be easily prepared for the realization of electrochemical sensors [15,39-42]. On the other hand, transition Metal Dichalcogenides (TMDs) materials have become an attractive alternative for electrochemical applications [43]. Layered transition-metal dichalcogenides, in particular, $\mathrm{WS}_{2}$ and $\mathrm{MoS}_{2}$, have attracted increasing interest as modifiers of working surfaces for hydrogen evolution reaction (HER) $[44,45]$ and energy storage [46-48]. The use of $\mathrm{MoS}_{2}$ in electrochemical sensing is a growing field and holds great promise in sensors and biosensors development [49-51]. However, despite the carbon black have been used for the construction of some electrodes for the direct polyphenol detections [15,52], even in foods matrices as olive oil[53], the potential of the use of $\mathrm{MoS}_{2}$ in the direct detection of polyphenols has not been yet exploited; just a paper reported on the $\mathrm{MoS}_{2}$ as electrode modifier for laccase biosensor used for red wine polyphenols indirect estimation [54].

In the last years $\mathrm{MoS}_{2}$-based nanocomposites have attracted researchers' attention [49]; since some $\mathrm{MoS}_{2}$ hybrid materials have shown great versatility as advanced electrode modifier, showing unique and sometimes unexpected sensing properties and catalytic activity [51,55,56]. The integration of carbon-based NMs [54,57], metal [58] or metal oxide nanoparticles [56] in $\mathrm{MoS}_{2}$ structures has been reported to have synergistic effects, exploitable in the electrochemical sensing.

The present paper describes, for the first time, the catechins determination ability on a new $\mathrm{CB} / \mathrm{MoS}_{2}$ nanohybrid electrode $(\mathrm{SPE}$ CB-MoS$)$. The analytical performance has been tested together with the regenerability of the sensor. The developed 
sensor was then applied to the assessment of the catechins content of 59 cocoa powder samples, expressed as epicatechin equivalent. The results obtained for the 59 cocoa powder samples correlated well with classical methods as FolinCiocalteu (FC), ABTS and gold nanoparticles-based (AuNPs) assay.

\section{Materials and methods}

\subsection{Reagents and stock solutions}

All the chemicals were of analytical grade. $\mathrm{MoS}_{2}$, Epicatechin, catechin, epigallocatechin, cetyltrimethylammonium chloride (CTAC; $25 \%$ in water), hydrogen tetracholoroaurate $\left(\mathrm{HAuCl}_{4} \cdot 3 \mathrm{H}_{2} \mathrm{O}\right.$, 99.9\%), 2,2-azino-bis(3ethylbenzothiazoline-6-sulphonic acid) (ABTS), sodium carbonate $\left(\mathrm{Na}_{2} \mathrm{CO}_{3}\right)$, Folin \& Ciocalteu's phenol reagent, sodium phosphate monobasic monohydrate ACS reagent $\left(\mathrm{NaH}_{2} \mathrm{PO}_{4} \cdot \mathrm{H}_{2} \mathrm{O}\right)$ sodium phosphate dibasic anhydrous $\left(\mathrm{Na}_{2} \mathrm{HPO}_{4}\right)$, potassium ferrocyanide, potassium ferricyanide, potassium chloride, methanol, N, N-dimethylformamide, dimethyl sulfoxide (DMSO), acetonitrile, acetic acid, sodium acetate, formic acid were purchased from Sigma Aldrich (St Louis, MO, USA). CB N220 was obtained from Cabot Corporation (Ravenna, Italy). Polyphenol stock solutions were prepared in methanol at a concentration of $1.0 \times 10^{-2} \mathrm{~mol} \mathrm{~L} \mathrm{~L}^{-1}$ and stored in the dark at $-20^{\circ} \mathrm{C}$. Milli-Q water $(18.2 \mathrm{M} \Omega)$ was employed for all the experiments.

\subsection{Apparatus}

Electrochemical measurements were carried out using an Autolab PGSTAT 12 potentiostat from Metrohm (Utrecht, Netherlands), screen-printed electrodes (SPE) were purchased from Dropsens S.L. (ref. DS110). Samples were shaken and centrifuged with an SSL1 orbital shaker from Stuart equipment (Belfast, UK) and an ALC4237R refrigerated centrifuge (ALC Intl., Cologno Monzese, Italy), respectively. For the gold nanoparticles assay, the reaction was performed in a water bath using a $720 \mathrm{D}$ thermostat digital group (Asal, Italy). Absorbance was recorded using a JENWAY 6400 spectrophotometer from Barlworld Scientific (Staffordshire, UK,).

\subsection{Samples}

A large number $\left(\mathrm{n}^{\circ}=59\right)$ of cocoa powder commercially available samples were analyzed. The investigated samples, coming from different varieties of cocoa and subjected to different production processes, were codified with numbers from 1 to 59 for reasons of corporate confidentiality. The samples were stored in plastic bags with pressure closure and kept in a cool, dry and dark place to avoid degradation phenomena.

In order to speed up the analysis, cocoa catechins were directly solubilized in DMSO, according to Della Pelle et al. 2015 [9], with some modifications. Briefly, $0.1 \mathrm{~g}$ of cocoa powder were weighted and solubilized in $1.5 \mathrm{~mL}$ of DMSO. The dispersion was vortexed for $1 \mathrm{~min}$ and sonicated in an ultrasonic bath for $5 \mathrm{~min}$ at a temperature of $20^{\circ} \mathrm{C}$. Subsequently, the dispersion was centrifuged at $12000 \mathrm{rpm}$ for 5 minutes at a temperature of $+4^{\circ} \mathrm{C}$ for $10 \mathrm{~min}$. The resulting supernatant was recovered and stored at $-20^{\circ} \mathrm{C}$ in the dark until analysis. In order to prove the effectiveness, of the proposed sample pretreatment, the samples have been extracted even according to $\mathrm{Gu}$ et al. [59], in this case, the defatting step [8] is followed by a solid-liquid extraction [59]. Both extracts, prior to each further analysis, were diluted to the appropriate dilution in $0.01 \mathrm{~mol} \mathrm{~L}^{-1}(\mathrm{pH} 7.0)$ phosphate buffer (PB).

\subsection{Preparation of the SPE-CB/MoS2}


$\mathrm{CB}$ and $\mathrm{MoS}_{2}$ dispersion volume, ratio/concentration and solvent were optimized in order to obtain the most effective electrodes in terms of sensitivity and reproducibility. The $1 \mathrm{mg} \mathrm{mL}^{-1} \mathrm{CB}$ and $\mathrm{MoS}_{2}$ dispersions were separately prepared in DMF-H20 1:1 (v/v) using a bath sonicator (3000683 Ultrasons Selecta, Barcelona, Spain) for $5 \mathrm{~h}$ at a temperature below $20^{\circ} \mathrm{C}$. The CB-MoS 2 75:25 (v/v) dispersion was prepared by mixing $\mathrm{CB}$ and $\mathrm{MoS}_{2}$ dispersions, the mix was further sonicated for $1 \mathrm{~h}$. This dispersion results usable for 1 month, however, before the use it requires a further 1 hour of sonication. This solution was employed for the SPEs modification, performed via drop casting onto the working electrode surface in five steps, for a total of $10 \mu \mathrm{L}$ allowing drying and solvent evaporation after each deposition, the latter process was sped up thanks to a warm light lamp. Before use, the electrodes were pretreated using $\mathrm{CV}$ (10 scans) in $0.1 \mathrm{mmol} \mathrm{L}^{-}$ ${ }^{1} \mathrm{~PB}(\mathrm{pH} 7)$ in $0.1 \mathrm{~mol} \mathrm{~L}^{-1} \mathrm{KCl}$, in the $-0.30 \mathrm{~V} /+0.7 \mathrm{~V}$ potential range (vs. pseudo- $\mathrm{Ag} / \mathrm{AgCl}$ ), using a scan rate of $0.5 \mathrm{~V}$ $\mathrm{s}^{-1}$. This procedure was also employed for the electrode surface regeneration, after the measurement of catechins standards or samples.

\subsection{Morphological characterization}

Field Emission Scanning Electron Microscopy (FE-SEM) was used to investigate the morphology of the SPE-CB/MoS 2 sensors. All the experiments were performed with a IIGMA high-resolution scanning electron microscope (Carl Zeiss Microscopy GmbH, Germany). Measurements were conducted on uncoated samples with an acceleration potential of 2 $\mathrm{kV}$ and at a working distance of about $4 \mathrm{~mm}$. All the micrographs were analysed with an image processing software (ImageJ, v.1.49.p) [60] in order to evaluate the mean dimension of the $\mathrm{MoS}_{2}$ crystals and the CB particles, together with the homogeneity of surface coverage and the relative percentage of $\mathrm{MoS}_{2}$ and $\mathrm{CB}$ in the SPE-CB/MoS electrode.

\subsection{Electrochemical measurements (CV, EIS, and DPV)}

The SPEs electrochemical behavior was investigated using CV and DPV; the measurements were carried out by deposition of $100 \mu \mathrm{L}$ of the analytes solution on the sensor surface. CVs of analytes were carried out using a scan rate of $0.050 \mathrm{~V} \mathrm{~s}^{-1}$ in the potential range $-0.20 \mathrm{~V} /+0.70 \mathrm{~V}$ (vs. pseudo $\mathrm{Ag} / \mathrm{AgCl}$ ) at different pHs: 4.0 and 5.0 in acetate buffer $\left(0.01 \mathrm{~mol} \mathrm{~L}^{-1}\right)$ and $\mathrm{pH}$ 6.0, 7.0, $8.0,9.0$ and 10.0 in phosphate buffer $\left(0.01 \mathrm{~mol} \mathrm{~L}^{-1}\right)$. The electrode passivation, after catechins analysis, was further assessed by electrochemical impedance spectroscopy (EIS) using $5 \mathrm{mmol} \mathrm{L}^{-1} \mathrm{Fe}(\mathrm{CN})_{6}{ }^{4-}$ 13- in $0.1 \mathrm{M} \mathrm{KCl}$. A sinusoidal wave with an amplitude of $\pm 10 \mathrm{mV}$ was employed, in a frequency scan range $10^{-2}-10^{5} \mathrm{~Hz}$ at open circuit potential, the results were analyzed by Nyquist plot (-Zre vs. Zim). DPV was used to quantify the analytes, the best DPV conditions were found using the simplex method, with the final aim to maximize the signal/noise ratio. DPVs were performed in phosphate $0.01 \mathrm{~mol} \mathrm{~L}^{-1} \mathrm{~PB}$ ( $\mathrm{pH} 7.0$ ), with a potential range of -0.15 to $+0.35 \mathrm{~V}$ with a pulse width of $50 \mathrm{~ms}$, and the final DPV pulse amplitude and scan rate were $20 \mathrm{mV}$ and $50 \mathrm{mV} \mathrm{s}^{-1}$, respectively. Before the use, the electrodes were pretreated with CVs (5 scans) in $0.1 \mathrm{mmol} \mathrm{L}^{-1} \mathrm{~PB}(\mathrm{pH} 7)$ in $0.1 \mathrm{~mol} \mathrm{~L}^{-1} \mathrm{KCl}$, in the potential range of $-0.30 \mathrm{~V}$ and $+0.7 \mathrm{~V}$ (vs. pseudo- $\mathrm{Ag} / \mathrm{AgCl}$ ), using a scan rate of $0.5 \mathrm{~V} \mathrm{~s}^{-1}$. The latter procedure was also employed for the electrode surface regeneration, after the measurement of catechins standards or samples.

\subsection{Methods comparison}

In order to compare the data obtained with the proposed sensor, the 59 cocoa powder samples were analyzed with three conventional methods for the polyphenols evaluations: ABTS, F.C., and AuNPs. Epicatechin standard solutions were used as reference standard to calibrate the methods. For each method, the possible DMSO reactivity/perturbation has been 
evaluated, however, there were no significant changes with respect to the aqueous solvent (at the dilution employed for the sample analysis).

\subsubsection{Folin-Ciocalteu procedure}

$20 \mu \mathrm{L}$ of a properly diluted cocoa sample, extracted in the conventional way and with the DMSO strategy (section 2.3), was added to $20 \mu \mathrm{L}$ of Folin-Ciocalteu reagent and stirred for $3 \mathrm{~min}$. Then, $400 \mu \mathrm{L}$ of sodium carbonate $\left(\mathrm{Na}_{2} \mathrm{CO}_{3}, 7.5 \%\right)$ and deionized water were added up to the final volume of $1000 \mu \mathrm{L}$. The solution was stirred at room temperature for 60 min, in the dark, and the total polyphenol content was determined at $760 \mathrm{~nm}$.

\subsubsection{ABTS assay procedure}

ABTS reagent stock solution was prepared according to Re et al. 1999 [61], the resulting radical ABTS ${ }^{++}$solution was diluted to reach an absorbance, reading at $734 \mathrm{~nm}$, of $0.70( \pm 0.02)$. An appropriate diluted cocoa extract volume, extracted in the conventional way and with the DMSO strategy (section 2.3), was added to the ABTS reagent (final volume 2000 $\mu \mathrm{L}$ ). Then the reaction mix was stored at room temperature for $5 \mathrm{~min}$, in the dark, to evaluate the radical scavenging activity. The absorbance value at $734 \mathrm{~nm}$ was recorded and the sample mediated ABTS + shutdown was evaluated with respect to the blank/control, prepared without the sample addition (using the same amount and type of solvent used for extraction) with the following equation: absorbance decrease $(\%)=[$ (control absorbance value - sample absorbance value)/ control absorbance value]x100.

\subsubsection{Gold nanoparticles assay procedure}

AuNPs based colorimetric assay was used for the total polyphenols determination according to Della Pelle et al. 2015 [9]. For the AuNPs formation $30 \mu \mathrm{L}$ of DMSO-cocoa powder extract, in the appropriate dilution, was mixed with $210 \mu \mathrm{L}$ of DMSO and stirred for 1 min with an orbital shaker. Then, $25 \mu \mathrm{L}$ of $\mathrm{HAuCl}_{4}$ solution $\left(2.0 \times 10^{-2} \mathrm{~mol} \mathrm{~L}^{-1}\right)$ and $235 \mu \mathrm{L}$ of phosphate buffer solution ( $\mathrm{pH} 8.0 ; 1.0 \times 10^{-2} \mathrm{~mol} \mathrm{~L}^{-1)}$ were added (final volume $500 \mu \mathrm{L}$ ). The solution was mixed with an orbital shaker for $1 \mathrm{~min}$ and the reaction was started heating the reaction mix for $5 \mathrm{~min}$ at $45{ }^{\circ} \mathrm{C}$ in a water bath. Finally, the reaction was blocked at $-20^{\circ} \mathrm{C}$ for 5 min to allow measurements in series. The absorbance due to the AuNPs formation was recorded at $540 \mathrm{~nm}$ and evaluated after the blank subtraction (the blank was composed by reaction mix without sample).

\section{Results and discussion}

\subsection{Electrochemical behavior of flavanols on hybrid $\mathrm{CB} / \mathrm{MoS} \mathrm{S}_{2}$ transducers}

In order to study the $\mathrm{CB}, \mathrm{MoS}_{2}$ and the resulting $\mathrm{CB} / \mathrm{MoS}_{2}$ combination, different amounts of the NMs dispersions have been drop casted onto SPE (section 2.4). Initially, cyclic voltammetry (CV) has been employed to study the electrochemical behavior of the SPE, SPE-CB, SPE-MoS 2 and SPE-CB/MoS 2 electrodes. The attention was focused on the first Catechin (CT) electron transfer reaction that occurs at very low positive potentials because the relevant radical scavenging activity [25,53,62-65]; the CT whole electrochemical behavior is discussed in detail in the supplementary material (Fig.S1). In Fig. 1, the CV curves obtained with $50 \mu \mathrm{mol} \mathrm{L}^{-1}$ of catechin in $0.01 \mathrm{~mol} \mathrm{~L}^{-1} \mathrm{~PB}(\mathrm{pH} 7.0)$, at $50 \mathrm{mV}$ $\mathrm{s}^{-1}$ scan rate, have been reported together with the relative blank (PB), performed before and after the catechin scan. In order to confirm the irreversibility of the electrode passivation (or CT attachment), the CT scan was followed by a mild surface regeneration (section 2.5). Improved electrochemical performances, in term of peak intensity and reversibility, were obtained in all cases compared to bare SPE. 
The bare SPE (Fig. 1A) shows the worst response in term of oxidation currents $\left(i_{p, a}=0.84 \mu A\right)$ and peak separation $\left(\Delta E_{p}\right.$ $=147 \mathrm{mV}$ ) and an evident 'memory effect' due to analyte's polymerization (blue line) after the scan and the regeneration step (5 CV scan in PB). These results are in accordance with literature [26], and the CV and EIS of Fig. S1 where catechin strongly adsorbed on the electrode surface hindering the electronic transfer. In Fig. 1B is clearly showed that SPE-CB display a significant oxidation current increase $\left(i_{p}, a=4.40 \mu \mathrm{A}\right)$ lowering the oxidation potential of CT $\left(\mathrm{SPE}-\mathrm{CB} \mathrm{E}_{\mathrm{p}, \mathrm{a}}=142\right.$ $\mathrm{mV}$ vs $\left.. \mathrm{SPE} \mathrm{E}_{\mathrm{p}, \mathrm{a}}=198 \mathrm{mV}\right)$ and showing a lower peak-to-peak separation $\left(\Delta \mathrm{E}_{\mathrm{p}}=76 \mathrm{mV}\right)$. However, in this case, the CT seems to remain attached to the electrode preserving the electroactivity and showing a residual current of the $97 \%$ (blue line). Similar behavior has been already reported for NMs, particularly with NMs with oxidized moieties [24] as in the case of CB $[36,66]$. Indeed, is reported that carboxyl and/or other oxygen-containing groups can behave as nucleophiles against the o-quinone ring (as Michael acceptor), leading to bond formation between flavanol and surface active functional groups [24]. On the other hand, the $\mathrm{SPE}-\mathrm{MoS}_{2}$ (Fig. 1C) displays just a slight increase of both oxidation peak $\left(\mathrm{i}_{\mathrm{p}, \mathrm{a}}=0.97\right.$ $\mu \mathrm{A})$ and reversibility $\left(\Delta \mathrm{E}_{\mathrm{p}}=88 \mathrm{mV}\right)$ compared with the bare SPE, this because the $\mathrm{MoS}_{2}$ has a low intrinsic conductivity $[44,46,57]$. However, is noteworthy the total absence of residual signal (blue line) after the CT scan, this $\mathrm{MoS}_{2}$ feature, at the best of our knowledge, has not been explored yet and could open new gates to $\mathrm{MoS}_{2}$ application as an antifouling functional material. Fig. 1D reports the SPE-CB/MoS 2 ability to merge the behavior of both materials. In fact, a significant enhancement of the analytical performance, compared to both the bare (SPE) and the single-NMs modified electrodes (SPE-CB and SPE-MoS $)_{2}$ is showed. The ratio CB-MoS $25: 25$ (v/v) (from now this electrode will be named SPE$\mathrm{CB} / \mathrm{MoS}_{2}$ ) was chosen, because higher amounts of $\mathrm{MoS}_{2}(50$ and $75 \%$, v/v) exhibited lower electrochemical improvements, probably due to $\mathrm{MoS}_{2}$ intrinsic low conductivity [44,46,57]. The SPE-CB/MoS displays a significant increase in the oxidation current $\left(\mathrm{i}_{\mathrm{p}, \mathrm{a}}=9.7 \mu \mathrm{A}\right)$ that occurs at lower potentials $\left(\mathrm{E}_{\mathrm{p}, \mathrm{a}}=98 \mathrm{mV}\right)$. Moreover, a significant decrease in the $\Delta \mathrm{E}_{\mathrm{p}}(22 \mathrm{mV})$ was observed. Considering the obtained $\Delta \mathrm{E}_{\mathrm{p}}$ (near to $30 \mathrm{mV}$ ) the reaction involves two electrons that can be attributed to the 'catecholic ring' [25]. The $\mathrm{pH}$ effect was also studied in Fig. $\mathbf{S 2}$ performing $\mathrm{CV}$ in a solution containing $25 \mu \mathrm{mol} \mathrm{L} \mathrm{L}^{-1}$ of CT. The peak intensities obtained at different $\mathrm{pH}$ catechin gave a straight line in the 4-8 $\mathrm{pH}$ range with a slope of $57 \mathrm{mV} \mathrm{pH}^{-1}$ unit. Considering these data, we can confirm a fast electron transfer with the same number of protons involved in the electrode reaction. The SPE-CB/MoS 2 modification performed with the $75 \%$ of $\mathrm{CB}$ and the $25 \%$ of $\mathrm{MoS}_{2}$ was able to totally prevent both the CT irreversible polymerization and the attachment onto the electrode surface. This behavior was confirmed in Fig. S3, where the EIS of the SPE-CB/MoS does not show any significant differences in the Nyquist plot after 4 consecutive scans of CT, confirming that the charge-transfer ability of the hybrid nanomaterial is not affected. In addition to the excellent electrochemical performance, a remarkable interelectrode reproducibility was obtained in terms of $\mathrm{i}_{\mathrm{p}, \mathrm{a}}$ and $\mathrm{E}_{\mathrm{p}, \mathrm{a}}$ for the CT oxidation peak (RSD 1.2\% and 3.5\%, respectively, n=10). Fig. 2 and Fig. S4 shows the SEM micrographs of the investigated electrodes. The SPE-MoS 2 (Fig.S4) is characterized by $\mathrm{MoS}_{2}$ 'crystals' with a broad size distribution centred around $870 \pm 550 \mathrm{~nm}$ (see particle size distribution in Fig. S5), composed by layers of stacked $\mathrm{MoS}_{2}$ flakes, with the nanometric uniform side (see Fig. S4 SPE$\mathrm{MoS}_{2}$ magnification). In the micrograph of the SPE-CB (Fig. S4) it is clearly visible the presence of CB primary units with a mean diameter of $20 \pm 8 \mathrm{~nm}$ (the particle size distribution is reported in Fig. S5) [67,68]. The SPE-CB/MoS 2 (Fig. 2) shows the co-localization of $\mathrm{MoS}_{2}$ 'crystals' and carbon black primary particles; the two components interact but maintain their original morphology. The CB intercalation seems to reduce the $\mathrm{MoS}_{2}$ natural tendency to restacking, with respect to the SPE-MoS , resulting in CB decorated $\mathrm{MoS}_{2}$ flakes. In particular, the $\sim 48 \%$ of the electrode surface is composed of $\mathrm{MoS}_{2}$ 'crystals' despite CB is added in a higher amount during the preparation of the electrode. This behaviour can be ascribed to the flakes-like conformation of $\mathrm{MoS}_{2}$ that favours their floating to the surface. The homogeneity of the obtained surfaces at the nanoscale was further evaluated by measuring the lacunarity of SEM 
micrographs (see figure S6). As expected, the SPE-CB electrode results in more homogeneous surface at the nanometric level, while the lacunarity associated to the SPE-MoS 2 system is higher, in accordance with the broad size distribution of the $\mathrm{MoS}_{2}$ crystals. Hence, as expected, the SPE-CB/MoS 2 presents an intermediate value of lacunarity with respect to the other two electrodes (SPE-CB and SPE-MoS 2 ), according to the co-presence of both CB and $\mathrm{MoS}_{2}$ morphologies.

In order to assess the exploitability of the SPE-CB/MoS for cocoa polyphenols analysis the proposed electrode has been tested against epicatechin (EP) and epigallocatechin (EG) (Fig. 3). These flavan-3-ols compounds, together with catechin, are the most abundant polyphenolic compounds of cocoa and chocolate and represent the monomers of the procyanidins $[3,69]$.

The SPE-CB/MoS 2 again showed good electrochemical performance despite the EP and EG different chemical structure (see Fig. 3 insets), resulting in different electrochemical behavior. In Fig. 3A it is clearly noticed the EP increased oxidation current $\left(\mathrm{i}_{\mathrm{p}, \mathrm{a}}=10 \mu \mathrm{A}\right)$ and the negative shift $\left(\mathrm{E}_{\mathrm{p}, \mathrm{a}}=88 \mathrm{mV}\right)$ in the oxidation potential with respect to the bare SPE $\left(\mathrm{i}_{\mathrm{p}, \mathrm{a}}=1.1 \mu \mathrm{A}, \mathrm{E}_{\mathrm{p}, \mathrm{a}}=198 \mathrm{mV}\right)$. Moreover, in the case of EP $(\mathbf{F i g . ~ 3 A})$, increased reversibility in terms of both peak intensity ratio and peak-to-peak separation was even recorded $\left(\Delta \mathrm{E}_{\mathrm{p}}=19 \mathrm{mV}\right)$. The CVs of EG $(\mathbf{F i g}$. 3B $)$, despite the expected irreversible behaviour [70], allowed, also in this case, an $E_{p}$, negative shift $\left(S P E-C B / M o S_{2} E_{p}, a=69 \mathrm{mV}\right.$ vs SPE $E_{p, a}$ $=120 \mathrm{mV})$ and a significantly improved anodic peak intensity $\left(\mathrm{i}_{\mathrm{p}, \mathrm{a}}=9.1 \mu \mathrm{A}\right)$ compared to the bare $\mathrm{SPE}\left(\mathrm{i}_{\mathrm{p}, \mathrm{a}}=1.6 \mu \mathrm{A}\right)$. Even with EP and EG, the SPE-CB/MoS 2 totally prevent both the flavanols irreversible polymerization and the attachment to the electrode surface. In addition, despite the different structures of CT, EP and EG a very close oxidation current and oxidation potential have been obtained for all the compounds.

\subsection{Analytical performance of hybrid $\mathrm{CB} / \mathrm{MoS}_{2}$ transducers for catechins determination}

The analytical performance of the SPE-CB/MoS 2 sensor for quantitative analysis was carefully evaluated by studying the response toward CT, EP and EG using differential pulse voltammetry (DPV) under optimized parameters (see section 2.5). Fig. 4 shows the voltammograms and the calibration curves obtained for the assayed concentrations of EP (A and D), CT (B and E) and EG (C and F) using the SPE-CB/MoS 2 . Analytical figures of merit for the SPE-CB/MoS 2 towards CT, EP and EG detection are listed in Table 1.

Table 1. Analytical characteristics of the SPE-CB/MoS 2 sensor employed for CT, EP, and EG detection.

\begin{tabular}{ccccc}
\hline \multirow{2}{*}{ Analyte } & $\begin{array}{c}\text { Equation } \\
(\mathrm{x}=\mu \mathrm{mol} \mathrm{L}-1 ; \mathrm{y}=\mu \mathrm{A})\end{array}$ & $\begin{array}{c}\text { Linear Range } \\
(\mu \mathrm{mol} \mathrm{L})\end{array}$ & $\begin{array}{c}\text { Sensitivity } \\
\left(\mu \mathrm{A} \mathrm{L}^{-1} \mathrm{~mol}^{-1}\right)\end{array}$ \\
\hline CT & $\mathrm{y}=1.122 \mathrm{x}+0.413$ & $0.1-25$ & 0.998 & 8.93 \\
EP & $\mathrm{y}=1.185 \mathrm{x}+0.762$ & $0.1-25$ & 0.998 & 0.18 \\
EG & $\mathrm{y}=1.092 \mathrm{x}+0.090$ & $0.1-25$ & 0.998 & 8.69 \\
\hline
\end{tabular}

${ }^{1}$ LODs were calculated as $3 \sigma /$ slope ratio, where $\sigma$ is the standard deviation of the mean value for 10 voltammograms of the blank. Analytical characteristics calculated using the mean value of three calibration curves.

Satisfactory linear range, sensitivity, and limit of detection were achieved in all the cases. Interestingly, all these analytical parameters are similar among them confirming the same anodic reactivity for the three analytes allowing the quantification of the flavanols index content with reliability. In addition, intra-electrode repeatability has been further confirmed by the RSD obtained with twenty different measurements performed with a solution containing $10 \mu$ mol $\mathrm{L}^{-1}$ epicatechin: Ip,a $<1.1 \%$ and Ep,a $<3.2$. The 'poor linearity' (Fig. 4 red curves) obtained for the bare SPE $\left(R^{2}=0.9406\right.$ $-0.7316)$ is in accordance with the $E_{p, a}$ positive shift $\left(E_{p, a}\right.$, average shift of $\left.200 \mathrm{mV}\right)$, that occurs for all the analytes, 
indicating an increase of the electrode surface passivation. Moreover, the lower sensitivity (about 2 orders of magnitude vs SPE-CB/MoS 2 ) of the bare SPE towards all the analytes is evident (Fig. 4).

Table 2 lists the performance of the CB-SPE/MoS 2 compared with other sensors and nanomaterial-based sensors for catechin detection.

Table 2. Analytical characteristics and application of electrochemical sensors employed for catechins detection.

\begin{tabular}{|c|c|c|c|c|c|c|}
\hline $\begin{array}{l}\text { Electrode / } \\
\text { Detection } \\
\text { Mechanism }\end{array}$ & $\begin{array}{c}\text { Food Matrix } \\
\text { (number of } \\
\text { samples) }\end{array}$ & $\begin{array}{c}\text { Sample } \\
\text { Pretreatment }\end{array}$ & Comparison methods & $\begin{array}{l}\text { Linear Range } \\
\left.\qquad(\mu \mathrm{mol} \mathrm{L})^{-1}\right)\end{array}$ & $\begin{array}{c}\text { LOD } \\
\left(\mu \mathrm{mol} \mathrm{L}{ }^{-1}\right)\end{array}$ & Ref. \\
\hline GCE/CV & $\begin{array}{l}\text { Cocoa powder, } \\
\text { chocolate }(n=19)\end{array}$ & $\begin{array}{l}\text { Defatting/Liquid } \\
\text { solid extraction }\end{array}$ & $\begin{array}{l}\text { F.C., total flavonoid, } \\
\text { DPPH, ABTS, FRAP }\end{array}$ & - & - & [38] \\
\hline Pt-PEDOT/DPV & $\begin{array}{l}\text { cocoa-based biscuit } \\
\qquad(\mathrm{n}=3)\end{array}$ & $\begin{array}{c}\text { Stirring /filtration on } \\
\text { Buchner }\end{array}$ & - & $0.7-8.6 *$ & $0.31 *$ & {$[35]$} \\
\hline $\begin{array}{l}\text { GR- thermally } \\
\text { reduced/DPV }\end{array}$ & $\operatorname{Beer}(n=3)$ & Dilution & - & $1.2-12.0^{* *}$ & - & [33] \\
\hline $\begin{array}{l}\text { GCE-N-doped } \\
\text { graphene/DPV }\end{array}$ & $\begin{array}{l}\text { Chinese green tea } \\
\qquad(\mathrm{n}=4)\end{array}$ & Dilution & - & $1.0-30 * *$ & $0.10 * *$ & {$[32]$} \\
\hline $\begin{array}{c}\text { SPE- } \\
\mathrm{CB} / \mathrm{MoS}_{2} / \mathrm{DPV}\end{array}$ & $\begin{array}{l}\text { Cocoa powder } \\
\quad(\mathrm{n}=59)\end{array}$ & $\begin{array}{c}\text { DMSO } \\
\text { solubilization }\end{array}$ & F.C., ABTS, AuNPs & $0.1-25.0 *$ & $0.17 *$ & [this work] \\
\hline
\end{tabular}

In this work, the obtained LODs and linear ranges are comparable or better than those reported in the literature, even when applied to other food matrices. It should be emphasized that among the reported works for the analysis of catechin, this is the only case using screen-printed technology. Importantly, no one of the reported works pays attention to the fouling process, that is the principal drawback for the catechin detection. In this study, the attention has been paid not only to the proposed sensor performances but even to the success and the exploitability of the application in complex samples, using a significant number of samples and comparing the results with other well-established methods (section 3.3.3).

Recovery (Fig. 5A) and renewability/regenerability (Fig. 5B) studies were also evaluated using a representative mix of cocoa powder samples with different polyphenol content (accordingly section 2.3 ).

The voltammograms obtained for the mix of samples (Fig 5A, blue line), and the fortified samples (Fig. 5A, green and red lines), exhibited identical $\mathrm{E}_{\mathrm{p}, \mathrm{a}},(90 \pm 5 \mathrm{mV})$ and excellent $\mathrm{RSD}$ for $\mathrm{i}_{\mathrm{p}, \mathrm{a}} \leq 1.2 \%,(\mathrm{n}=3)$ as well as quantitative recoveries with values ranging from $94 \%$ to $103 \%$ demonstrating the sensor exploitability for the catechins determination in complex samples.

Fig. 5B shows the voltammograms obtained with cocoa samples with low (sample 12, green line), medium (sample 5, black line) and high (sample 1, blue line) polyphenols content (each diluted at the adequate dilution to fit the signal in the calibration plot). Noteworthy is the very high regenerability showed by the SPE-CB/MoS . This behavior was proven by monitoring the electrode state during the 59 sample measurements (interspersed with a fast surface regeneration see section 2.5). The SPE-CB/MoS 2 regenerability is further underlined in Fig. $\mathbf{5 B}$, where the dashed red line represents the Sample 5 measured after measuring the whole set of samples, obtaining a signal recovery of $99 \%$. These results are in accordance with previously discussed results (see section 3.1) and confirm the ability of the SPE-CB/MoS 2 to avoid the permanent catechins polymerization or attachment onto the electrode surface. 
To prove the sensor surface regenerability, the whole set of samples (59 cocoa powders) has been measured on the same SPE-CB/MoS 2 , and the electrode 'state' has been monitored measuring epicatechin standard $\left(10 \mu \mathrm{mol} \mathrm{L}^{-1}\right)$ every 5 samples measured (Fig. 5C). Impressively, Figure 5C, shows a very stable response obtained that result in RSD of the signal obtained of $0.9 \%$ and $5,2 \%$ for the $\mathrm{I}_{\mathrm{p}}$, a and $\mathrm{E}_{\mathrm{p}}, \mathrm{a}$, respectively.

\subsection{Quantitative sample analysis of total catechins on hybrid $\mathrm{CB} / \mathrm{MoS} \mathrm{S}_{2}$ transducers}

Then the applicability of the CB-SPE/MoS for the determination of flavanols was rigorously carried out through the analysis of a wide set of cocoa samples $(n=59)$. Table 3 lists the quantitative analysis of cocoa samples by the proposed $\mathrm{SPE}-\mathrm{CB} / \mathrm{MoS}_{2}$ sensor. In order to demonstrate analytical reliability of the proposed electrochemical sensor, cocoa powder samples were also analyzed by the classical F.C. (total polyphenols) and ABTS (antioxidant capacity) assays as well as by AuNPs-based free-extraction approach [59]. Particular attention was paid on the AuNPs method, which relies on the direct formation of AuNPs in organic solvent (DMSO)-aqueous medium, driven by endogenous polyphenols. This 'extraction-free' approach involves the use of DMSO acting as an organic solvent, able to 'interact', at the same time, with the fatty matrix and with the aqueous medium of measurement, making the cocoa polyphenols analysis faster and easier. The effectiveness of this DMSO-based approach was tested with the set of 59 cocoa samples and compared both with a conventional liquid-liquid extraction (LL) methods which need a defatting step [59] (section 2.3) and with the well-established methods of Folin-Ciocalteu and ABTS. The effectiveness of the proposed DMSO-based approach was confirmed by the good correlations (Fig. S7) obtained between DMSO-based and the LL conventional method (F-C: r=0.954 and ABTS: $r=0.971, n=59$ ) confirming the suitability of the DMSO-based extraction-free approach. Furthermore, the DMSO-based approach requires a lower amount of sample $(0.1 \mathrm{~g}$ vs. $8.0 \mathrm{~g})$, a significant lower solvent consumption occurs (1.5 mL of DMSO vs. $150 \mathrm{~mL}$ of hexane and $5 \mathrm{~mL}$ of $5 \mathrm{~mL}$ of 70:29.5:0.5 acetone/water/acetic acid) as well as a reduction in waste generation; moreover, the procedure is not time-consuming (15' min vs. $8 \mathrm{~h}$ ). Therefore, the DMSObased free-extraction approach was selected for the quantitative determination of polyphenols in cocoa samples using the proposed SPE-CB/MoS 2 electrochemical sensor (see Table 3).

Table 3. Analysis of cocoa samples by the SPE-CB/MoS 2 and the F.C, ABTS and AuNPs assays ${ }^{1}$.

\begin{tabular}{|c|c|c|c|c|c|c|c|c|c|c|c|c|}
\hline \multirow{2}{*}{$\begin{array}{c}\text { Sample } \\
1\end{array}$} & \multicolumn{3}{|c|}{$\begin{array}{c}\text { SPE-CB/MoS } \\
\left(\mathrm{mg} \mathrm{g}^{-1}\right)\end{array}$} & \multicolumn{3}{|c|}{$\begin{array}{c}\text { Folin-Ciocalteu } \\
\left(\mathrm{mg} \mathrm{g}^{-1}\right)\end{array}$} & \multicolumn{3}{|c|}{$\begin{array}{c}\text { ABTS } \\
\left(\mathrm{mg} \mathrm{g}^{-1}\right)\end{array}$} & \multicolumn{3}{|c|}{$\begin{array}{l}\text { AuNPs } \\
\left(\mathrm{mg} \mathrm{g}^{-1}\right)\end{array}$} \\
\hline & 86.1 & \pm & 0.3 & 86.0 & \pm & 0.5 & 72.5 & \pm & 0.3 & 86.0 & \pm & 4.8 \\
\hline 2 & 50.4 & \pm & 0.6 & 45.3 & \pm & 0.5 & 40.2 & \pm & 0.3 & 49.2 & \pm & 0.3 \\
\hline 3 & 34.1 & \pm & 0.3 & 31.2 & \pm & 1.6 & 28.2 & \pm & 1.2 & 37.2 & \pm & 0.2 \\
\hline 4 & 59.9 & \pm & 0.5 & 48.7 & \pm & 0.2 & 37.6 & \pm & 2.4 & 47.5 & \pm & 0.1 \\
\hline 5 & 39.2 & \pm & 0.5 & 32.2 & \pm & 2.2 & 22.6 & \pm & 0.6 & 29.7 & \pm & 0.6 \\
\hline 6 & 55.1 & \pm & 1.3 & 49.2 & \pm & 2.3 & 42.1 & \pm & 1.1 & 50.1 & \pm & 4.4 \\
\hline 7 & 37.9 & \pm & 0.4 & 27.7 & \pm & 2.1 & 21.7 & \pm & 0.2 & 30.9 & \pm & 3.7 \\
\hline 8 & 58.4 & \pm & 1.4 & 52.3 & \pm & 2.3 & 39.3 & \pm & 1.8 & 52.2 & \pm & 4.3 \\
\hline 9 & 36.7 & \pm & 1.1 & 30.1 & \pm & 1.1 & 25.0 & \pm & 1.1 & 32.0 & \pm & 1.8 \\
\hline 10 & 26.6 & \pm & 1.1 & 17.4 & \pm & 0.7 & 186 & \pm & 1.0 & 26.3 & \pm & 1.8 \\
\hline 11 & 31.9 & \pm & 0.5 & 19.2 & \pm & 0.7 & 21.2 & \pm & 0.3 & 23.9 & \pm & 1.3 \\
\hline 12 & 26.0 & \pm & 1.0 & 16.0 & \pm & 0.6 & 18.7 & \pm & 0.4 & 20.2 & \pm & 0.6 \\
\hline 13 & 14.5 & \pm & 0.4 & 3.7 & \pm & 0.1 & 4.1 & \pm & 0.1 & 11.5 & \pm & 0.1 \\
\hline 14 & 14.4 & \pm & 0.5 & 3.4 & \pm & 0.4 & 3.6 & \pm & 0.5 & 11.5 & \pm & 0.3 \\
\hline 15 & 23.2 & \pm & 0.6 & 16.2 & \pm & 1.1 & 17.9 & \pm & 0.3 & 20.3 & \pm & 0.9 \\
\hline 16 & 28.0 & \pm & 0.4 & 20.0 & \pm & 1.9 & 20.3 & \pm & 0.3 & 25.1 & \pm & 1.0 \\
\hline 17 & 21.9 & \pm & 1.0 & 8.8 & \pm & 0.2 & 9.3 & \pm & 0.1 & 16.7 & \pm & 1.2 \\
\hline 18 & 25.0 & \pm & 0.6 & 12.5 & \pm & 0.5 & 9.4 & \pm & 0.9 & 19.9 & \pm & 0.9 \\
\hline 19 & 15.7 & \pm & 0.3 & 3.4 & \pm & 0.1 & 3.1 & \pm & 0.5 & 11.0 & \pm & 0.6 \\
\hline 20 & 22.2 & \pm & 0.6 & 11.1 & \pm & 0.1 & 10.8 & \pm & 0.9 & 18.3 & \pm & 0.4 \\
\hline 21 & 15.0 & \pm & 0.6 & 4.4 & \pm & 0.1 & 3.2 & \pm & 0.1 & 11.5 & \pm & 0.4 \\
\hline
\end{tabular}




\begin{tabular}{|c|c|c|c|c|c|c|c|c|c|c|c|c|}
\hline 22 & 14.6 & \pm & 0.5 & 2.5 & \pm & 0.1 & 2.7 & \pm & 0.5 & 10.0 & \pm & 0.2 \\
\hline 23 & 14.5 & \pm & 0.3 & 2.7 & \pm & 0.1 & 3.0 & \pm & 0.1 & 10.6 & \pm & 1.2 \\
\hline 24 & 14.9 & \pm & 0.5 & 3.4 & \pm & 0.1 & 3.0 & \pm & 0.0 & 10.5 & \pm & 0.6 \\
\hline 25 & 29.0 & \pm & 0.9 & 20.7 & \pm & 0.7 & 21.4 & \pm & 2.0 & 22.3 & \pm & 3.1 \\
\hline 26 & 42.0 & \pm & 1.1 & 29.9 & \pm & 0.9 & 34.4 & \pm & 0.6 & 42.4 & \pm & 1.3 \\
\hline 27 & 27.3 & \pm & 0.7 & 24.2 & \pm & 0.1 & 21.4 & \pm & 2.0 & 24.7 & \pm & 0.8 \\
\hline 28 & 43.0 & \pm & 1.5 & 27.6 & \pm & 1.0 & 23.6 & \pm & 0.4 & 30.3 & \pm & 1.8 \\
\hline 29 & 31.6 & \pm & 0.8 & 20.1 & \pm & 0.1 & 18.5 & \pm & 0.8 & 24.0 & \pm & 0.3 \\
\hline 30 & 15.0 & \pm & 0.5 & 5.2 & \pm & 0.2 & 4.3 & \pm & 0.0 & 11.6 & \pm & 0.2 \\
\hline 31 & 16.1 & \pm & 0.4 & 5.6 & \pm & 0.3 & 4.9 & \pm & 0.6 & 13.1 & \pm & 0.7 \\
\hline 32 & 36.3 & \pm & 0.5 & 27.4 & \pm & 1.2 & 20.9 & \pm & 0.0 & 27.4 & \pm & 0.1 \\
\hline 33 & 41.6 & \pm & 0.1 & 25.8 & \pm & 0.3 & 22.8 & \pm & 0.1 & 28.5 & \pm & 0.9 \\
\hline 34 & 21.5 & \pm & 0.1 & 4.6 & \pm & 0.5 & 3.7 & \pm & 0.5 & 10.5 & \pm & 0.5 \\
\hline 35 & 31.2 & \pm & 0.3 & 13.2 & \pm & 1.0 & 15.3 & \pm & 0.8 & 23.1 & \pm & 1.7 \\
\hline 36 & 44.4 & \pm & 1.1 & 27.3 & \pm & 1.9 & 23.2 & \pm & 1.0 & 30.7 & \pm & 1.1 \\
\hline 37 & 31.3 & \pm & 0.8 & 22.7 & \pm & 0.9 & 16.3 & \pm & 0.1 & 25.7 & \pm & 0.4 \\
\hline 38 & 35.5 & \pm & 1.1 & 27.2 & \pm & 0.2 & 15.0 & \pm & 0.5 & 30.8 & \pm & 0.3 \\
\hline 39 & 31.9 & \pm & 0.4 & 17.3 & \pm & 0.7 & 23.3 & \pm & 0.1 & 32.4 & \pm & 4.2 \\
\hline 40 & 22.4 & \pm & 0.4 & 3.8 & \pm & 0.1 & 3.4 & \pm & 0.5 & 12.6 & \pm & 0.6 \\
\hline 41 & 21.8 & \pm & 0.1 & 7.8 & \pm & 0.2 & 5.0 & \pm & 0.1 & 12.4 & \pm & 0.3 \\
\hline 42 & 15.1 & \pm & 0.2 & 3.6 & \pm & 0.5 & 3.3 & \pm & 0.0 & 10.8 & \pm & 0.8 \\
\hline 43 & 22.7 & \pm & 0.5 & 5.7 & \pm & 0.2 & 4.3 & \pm & 0.1 & 13.4 & \pm & 1.5 \\
\hline 44 & 13.1 & \pm & 0.4 & 3.5 & \pm & 0.0 & 3.4 & \pm & 0.5 & 10.6 & \pm & 0.3 \\
\hline 45 & 31.0 & \pm & 0.1 & 29.1 & \pm & 0.1 & 29.7 & \pm & 0.9 & 34.0 & \pm & 2.0 \\
\hline 46 & 21.7 & \pm & 0.3 & 10.7 & \pm & 0.7 & 10.5 & \pm & 0.2 & 15.6 & \pm & 0.8 \\
\hline 47 & 21.7 & \pm & 0.7 & 10.1 & \pm & 0.4 & 13.8 & \pm & 0.1 & 18.5 & \pm & 1.9 \\
\hline 48 & 22.3 & \pm & 0.2 & 11.6 & \pm & 0.6 & 12.8 & \pm & 0.9 & 18.5 & \pm & 0.4 \\
\hline 49 & 55.2 & \pm & 1.4 & 47.2 & \pm & 3.9 & 38.6 & \pm & 1.9 & 45.8 & \pm & 0.3 \\
\hline 50 & 22.5 & \pm & 0.8 & 10.6 & \pm & 0.6 & 10.0 & \pm & 0.5 & 16.6 & \pm & 0.5 \\
\hline 51 & 32.6 & \pm & 1.0 & 18.3 & \pm & 2.0 & 27.3 & \pm & 0.7 & 25.8 & \pm & 0.2 \\
\hline 52 & 14.7 & \pm & 0.2 & 2.6 & \pm & 0.2 & 2.6 & \pm & 0.5 & 9.9 & \pm & 0.4 \\
\hline 53 & 19.7 & \pm & 0.5 & 5.9 & \pm & 0.2 & 3.2 & \pm & 0.5 & 12.7 & \pm & 1.0 \\
\hline 54 & 31.6 & \pm & 1.0 & 29.9 & \pm & 0.1 & 28.1 & \pm & 0.8 & 28.9 & \pm & 0.2 \\
\hline 55 & 59.0 & \pm & 0.4 & 42.5 & \pm & 0.1 & 38.2 & \pm & 0.6 & 44.6 & \pm & 0.3 \\
\hline 56 & 34.2 & \pm & 0.5 & 28.5 & \pm & 0.3 & 24.6 & \pm & 2.1 & 32.9 & \pm & 1.9 \\
\hline 57 & 49.2 & \pm & 1.4 & 37.8 & \pm & 0.1 & 30.9 & \pm & 0.5 & 42.2 & \pm & 1.2 \\
\hline 58 & 54.5 & \pm & 1.4 & 49.2 & \pm & 7.1 & 41.4 & \pm & 0.4 & 54.5 & \pm & 0.8 \\
\hline 59 & 43.4 & \pm & 0.5 & 35.6 & \pm & 2.6 & 30.0 & \pm & 0.5 & 35.6 & \pm & 1.6 \\
\hline
\end{tabular}

${ }^{1}$ The data are expressed as epicatechin equivalents as mean values $\pm \mathrm{SD}, \mathrm{n}=3$. Calibration curves (performed with epicatechin; $\mathrm{x}=\mu \mathrm{mol} \mathrm{L}-1$ and $\mathrm{y}=$ Abs.) showed both good linearity and coefficients of determination: F.C. $y=0.1587 x-0.0032, R^{2}=0.9971 ; A B T S y=69.682 x+0.7792, R^{2}=0.9925$; AuNPs y $=0.0291 \mathrm{x}-0.0753, \mathrm{R}^{2}=0.9940$.

Despite heterogeneity of the samples, in terms of cocoa types, fat content (5.6-26.1\%), color and polyphenols content, the data obtained with the SPE-CB/MoS 2 resulted highly correlated with all the well-established methods assays FC ( $\mathrm{r}=0.972$ ) and ABTS (r=0.966) as well as with the AuNPS-based (r=0.949).

\section{Conclusions}

Herein an electrochemical sensor based on the synergic employment of $\mathrm{MoS}_{2}$ and $\mathrm{CB}$ is successfully applied to catechins determination in complex cocoa samples. The SPE-CB/MoS 2 electrodes merge the $\mathrm{CB}$ ability to enhance the electrochemical response and the $\mathrm{MoS}_{2}$ 'antifouling' properties against catechins, drawbacks that occur to both conventional carbons and NMs-modified electrodes. To the best of our knowledge, this latter $\mathrm{MoS}_{2}$ hidden feature has been deeply studied and proven for the first time in this article. The realized sensor showed an impressive stable and reproducible response, with low working potentials, excellent resistance to fouling with detection limits in the nanomolar range. Noteworthily, the whole set of 59 samples have been measured using a single SPE-CB/MoS 2 , without loss of signal, proving it excellent 'antifouling' properties. Moreover, in order to speed up and simplify, the cocoa powder 
polyphenols analysis (that required $8 \mathrm{~h}$ ), a fast (15 min) direct cocoa polyphenols DMSO-based solubilization extractionfree strategy is proposed. Definitely, the proposed approach is clearly intended for fast (total time $15^{\prime}$ min, from sampling to analysis) evaluation of polyphenols in cocoa powder, the fast DMSO 'extraction' (15 min, without volatile solvents) together to the SPE-CB/MoS 2 (regenerable and portable) allows a real delocalization of the analysis and could be extended for the catechins analysis in other food matrices. The 'DMSO-based polyphenols approach results rapid, effective and eco-friendly (total working volume $1.5 \mathrm{~mL}$ ), does not require evaporation steps (and volatile compounds) and can be coupled with cocoa polyphenols (and antioxidant capacity) content evaluation methods. Definitely, the SPE-CB/MoS 2 showed an important synergistic effect and the combination of these nanomaterials can offer new opportunities to develop new devices and applications. In our case the proposed SPE-CB/MoS 2 overcome the proof of applicability, becoming an analytical tool able to overtake the classics catechins analytical drawbacks.

\section{Acknowledgments}

This work is supported by funding from the European Union's Horizon 2020 research and innovation programme under the Marie Skłodowska-Curie grant agreement No. 713714 and co-funding of University of Teramo and Abruzzo region. The authors acknowledge Professor Giampiero Sacchetti (University of Teramo, Department of Food Science) for the cocoa powder samples donation. The authors acknowledge Professor Emiliano Fratini (University of Florence, Department of Chemistry) to supervising the morphological analysis. 


\section{References}

[1] R. Tsao, Chemistry and biochemistry of dietary polyphenols, Nutrients. 2 (2010) 1231-1246. doi:10.3390/nu2121231.

[2] A. Belščak, D. Komes, D. Horžić, K.K. Ganić, D. Karlović, Comparative study of commercially available cocoa products in terms of their bioactive composition, Food Res. Int. 42 (2009) 707-716. doi:10.1016/j.foodres.2009.02.018.

[3] C. Di Mattia, G. Sacchetti, D. Mastrocola, M. Serafini, From cocoa to chocolate: The impact of processing on in vitro antioxidant activity and the effects of chocolate on antioxidant markers in vivo, Front. Immunol. 8 (2017) 1-7. doi:10.3389/fimmu.2017.01207.

[4] M.S. Beg, S. Ahmad, K. Jan, K. Bashir, Status, supply chain and processing of cocoa - A review, Trends Food Sci. Technol. 66 (2017) 108-116. doi:10.1016/j.tifs.2017.06.007.

[5] J.S. Bonvehi, F.V. Coil, Evaluation of bitterness and astringency of polyphenolic compounds in cocoa powder, Food Chem. 60 (1997) 365-370.

[6] C. Andres-Lacueva, M. Monagas, N. Khan, M. Izquterdo-Pulido, M. Urpi-Sarda, J. Permanyer, R.M. LamuelaRaventós, Flavanol and flavonol contents of cocoa powder products: Influence of the manufacturing process, J. Agric. Food Chem. 56 (2008) 3111-3117. doi:10.1021/jf0728754.

[7] F. Ioannone, C.D. Di Mattia, M. De Gregorio, M. Sergi, M. Serafini, G. Sacchetti, Flavanols, proanthocyanidins and antioxidant activity changes during cocoa (Theobroma cacao L.) roasting as affected by temperature and time of processing, Food Chem. 174 (2015) 256-262. doi:10.1016/j.foodchem.2014.11.019.

[8] C. Di Mattia, M. Martuscelli, G. Sacchetti, I. Scheirlinck, B. Beheydt, D. Mastrocola, P. Pittia, Effect of Fermentation and Drying on Procyanidins, Antiradical Activity and Reducing Properties of Cocoa Beans, Food Bioprocess Technol. 6 (2013) 3420-3432. doi:10.1007/s11947-012-1028-x.

[9] F. Della Pelle, M.C. González, M. Sergi, M. Del Carlo, D. Compagnone, A. Escarpa, Gold Nanoparticles-based Extraction-Free Colorimetric Assay in Organic Media: An Optical Index for Determination of Total Polyphenols in Fat-Rich Samples, Anal. Chem. 87 (2015) 6905-6911. doi:10.1021/acs.analchem.5b01489.

[10] I. Ignat, I. Volf, V.I. Popa, A critical review of methods for characterisation of polyphenolic compounds in fruits and vegetables, Food Chem. 126 (2011) 1821-1835. doi:10.1016/j.foodchem.2010.12.026.

[11] V. Ferrone, S. Genovese, M. Carlucci, M. Tiecco, R. Germani, F. Preziuso, F. Epifano, G. Carlucci, V.A. Taddeo, A green deep eutectic solvent dispersive liquid-liquid micro-extraction (DES-DLLME) for the UHPLC-PDA determination of oxyprenylated phenylpropanoids in olive, soy, peanuts, corn, and sunflower oil, Food Chem. 245 (2018) 578-585. doi:10.1016/j.foodchem.2017.10.135.

[12] R.V.S.S. Gottumukkala, N. Nadimpalli, K. Sukala, G. V Subbaraju, Determination of Catechin and Epicatechin Content in Chocolates by High-Performance Liquid Chromatography, Int. Sch. Res. Not. 2014 (2014). doi:10.1155/2014/628196.

[13] T.H. Stanley, A.T. Smithson, A.P. Neilson, R.C. Anantheswaran, J.D. Lambert, Analysis of Cocoa Proanthocyanidins Using Reversed Phase High- Performance Liquid Chromatography and Electrochemical Detection: Application to Studies on the E ff ect of Alkaline Processing, J. Agric. Food Chem. 63 (2015) 59705975. doi:10.1021/acs.jafc.5b02661.

[14] F. Blasi, G. Rocchetti, D. Montesano, L. Lucini, G. Chiodelli, S. Ghisoni, G. Baccolo, M.S. Simonetti, L. Cossignani, Changes in extra-virgin olive oil added with Lycium barbarum L. carotenoids during frying: Chemical analyses and metabolomic approach, Food Res. Int. 105 (2018) 507-516. doi:10.1016/j.foodres.2017.11.061.

[15] F. Della Pelle, D. Compagnone, Nanomaterial-based sensing and biosensing of phenolic compounds and related antioxidant capacity in food, Sensors. 18 (2018) 462. doi:10.3390/s18020462.

[16] J. Hoyos-arbeláez, M. Vázquez, J. Contreras-calderón, Electrochemical methods as a tool for determining the antioxidant capacity of food and beverages: A review, Food Chem. 221 (2017) 1371-1381. doi:10.1016/j.foodchem.2016.11.017.

[17] D.A. El-Hady, N. Abo El Maali, Determination of catechin isomers in human plasma subsequent to green tea ingestion using chiral capillary electrophoresis with a high-sensitivity cell, Talanta. 76 (2008) 138-145. doi:10.1016/j.talanta.2008.02.026.

[18] X. Huang, E. Teye, L.K. Sam-Amoah, F. Han, L. Yao, W. Tchabo, Rapid measurement of total polyphenols content in cocoa beans by data fusion of NIR spectroscopy and electronic tongue, Anal. Methods. 6 (2014) 5008-5015. doi:10.1039/c4ay00223g.

[19] Y. Hu, Z.J. Pan, W. Liao, J. Li, P. Gruget, D.D. Kitts, X. Lu, Determination of antioxidant capacity and phenolic content of chocolate by attenuated total reflectance-Fourier transformed-infrared spectroscopy, Food Chem. 202 (2016) 254-261. doi:10.1016/j.foodchem.2016.01.130.

[20] M.F. Barroso, C. Delerue-matos, M.B.P.P. Oliveira, Towards a reliable technology for antioxidant capacity and oxidative damage evaluation : Electrochemical ( bio ) sensors, Biosens. Bioelectron. 30 (2011) 1-12. doi:10.1016/j.bios.2011.08.036.

[21] R. Prehn, J. Gonzalo-Ruiz, M. Cortina-Puig, Electrochemical detection of polyphenolic compounds in foods 
D. Rojas, F. Della Pelle, E. Del Carlo, Michele Fratini, D. Escarpa, Alberto Compagnone, Nanohybrid carbon black-molybdenum disulfide transducers for preconcentration-free voltammetric detection of the olive oil odiphenols hydroxytyrosol and oleuropein, Microchim. Acta. (2019). doi:10.1007/s00604-019-3418-5.

[23] T.A. Enache, A.M. Oliveira-brett, Phenol and para -substituted phenols electrochemical oxidation pathways, J. Electroanal. Chem. 655 (2011) 9-16. doi:10.1016/j.jelechem.2011.02.022.

[24] M. Amiri-Aref, J.B. Raoof, R. Ojani, A highly sensitive electrochemical sensor for simultaneous voltammetric determination of noradrenaline, acetaminophen, xanthine and caffeine based on a flavonoid nanostructured modified glassy carbon electrode, Sensors Actuators, B Chem. 192 (2014) 634-641. doi:10.1016/j.snb.2013.11.006.

[25] P. Janeiro, A.M. Oliveira Brett, Catechin electrochemical oxidation mechanisms, Anal. Chim. Acta. 518 (2004) 109-115. doi:10.1016/j.aca.2004.05.038.

[26] L. Blandón-Naranjo, F. Della Pelle, M. V. Vázquez, J. Gallego, A. Santamaría, M. Alzate-Tobón, D. Compagnone, Electrochemical Behaviour of Microwave-assisted Oxidized MWCNTs Based Disposable Electrodes: Proposal of a NADH Electrochemical Sensor, Electroanalysis. 30 (2018) 509-516. doi:10.1002/elan.201700674.

[27] F.S. Ligler, H.S. White, Nanomaterials in analytical chemistry, Anal. Chem. 85 (2013) 11161-11162. doi:10.1021/ac403331m.

[28] M. Pumera, A. Escarpa, Nanomaterials as electrochemical detectors in microfluidics and CE fundamentals, designs, and applications, Electrophoresis. 30 (2009) 3315-3323. doi:https://doi.org/10.1002/elps.200900008.

[29] D. Capoferri, F. Della Pelle, M. Del Carlo, D. Compagnone, Affinity Sensing Strategies for the Detection of Pesticides in Food, Foods. 7 (2018) 148. doi:10.3390/foods7090148.

[30] F. Della Pelle, A. Scroccarello, S. Scarano, D. Compagnone, Silver nanoparticles-based plasmonic assay for the determination of sugar content in food matrices, Anal. Chim. Acta. (2018). doi:https://doi.org/10.1016/j.aca.2018.11.015.

[31] S. Masoum, M. Behpour, F. Azimi, M.H. Motaghedifard, Potentiality of chemometric approaches for the determination of $(+)$-catechin in green tea leaves at the surface of multiwalled carbon nanotube paste electrode, Sensors Actuators, B Chem. 193 (2014) 582-591. doi:10.1016/j.snb.2013.12.022.

[32] J. Pang, X. Wu, A. Li, X. Liu, M. Li, Detection of catechin in Chinese green teas at N-doped carbon-modified electrode, Ionics (Kiel). 23 (2017) 1889-1895. doi:10.1007/s11581-017-2006-0.

[33] C. Chu'Er, Z. Sofer, M. Pumera, A. Bonanni, Doped and undoped graphene platforms the influence of structural properties on the detection of polyphenols, Sci. Rep. 22 (2016) 3830-3834.

[34] V. Andrei, E. Sharpe, A. Vasilescu, S. Andreescu, A single use electrochemical sensor based on biomimetic nanoceria for the detection of wine antioxidants, Talanta. 156-157 (2016) 112-118. doi:10.1016/j.talanta.2016.04.067.

[35] L. Pigani, R. Seeber, A. Bedini, E. Dalcanale, M. Suman, Adsorptive-Stripping Voltammetry at PEDOTModified Electrodes. Determination of Epicatechin, Food Anal. Methods. 7 (2014) 754-760. doi:10.1007/s12161-013-9678-5.

[36] M. Gandhi, D. Rajagopal, S. Parthasarathy, S. Raja, S.T. Huang, A. Senthil Kumar, In Situ Immobilized Sesamol-Quinone/Carbon Nanoblack-Based Electrochemical Redox Platform for Efficient Bioelectrocatalytic and Immunosensor Applications, ACS Omega. 3 (2018) 10823-10835. doi:10.1021/acsomega.8b01296.

[37] G. Ziyatdinova, E. Kozlova, H. Budnikov, Polyquercetin/MWNT-modified Electrode for the Determination of Natural Phenolic Antioxidants, Electroanalysis. 29 (2017) 2610-2619. doi:10.1002/elan.201700440.

[38] J.M. Brcanović, A.N. Pavlović, S.S. Mitić, G.S. Stojanović, D.D. Manojlović, B.M. Kaličanin, J.N. Veljković, J.N. Veljkovi, S.S.M. Jelena M. Brcanovi, Aleksandra N. Pavlovi, G.S. Stojanovi, D.D. Manojlovi, B.M. Kalianin, Cyclic voltammetry determination of antioxidant capacity of cocoa powder, dark chocolate and milk chocolate samples: Correlation with spectrophotometric assays and individual phenolic compounds, Food Technol. Biotechnol. 51 (2013) 460-470. doi:10.1097/BTE.0000000000000074.

[39] M. Baccarin, F.A. Santos, F.C. Vicentini, V. Zucolotto, B.C. Janegitz, O. Fatibello-Filho, Electrochemical sensor based on reduced graphene oxide/carbon black/chitosan composite for the simultaneous determination of dopamine and paracetamol concentrations in urine samples, J. Electroanal. Chem. 799 (2017) 436-443. doi:10.1016/j.jelechem.2017.06.052.

[40] T.A. Silva, F.C. Moraes, B.C. Janegitz, O. Fatibello-Filho, Electrochemical Biosensors Based on Nanostructured Carbon Black: A Review, J. Nanomater. 2017 (2017) 1-14. doi:10.1155/2017/4571614.

[41] D. Rojas, F. Della Pelle, M. Del Carlo, M. D’Angelo, R. Dominguez-Benot, A. Cimini, A. Escarpa, D. Compagnone, Electrodeposited Prussian Blue on carbon black modified disposable electrodes for direct enzyme-free H2O2sensing in a Parkinson's disease in vitro model, Sensors Actuators, B Chem. 275 (2018) 402-408. doi:10.1016/j.snb.2018.08.040.

[42] F. Della Pelle, M. Del Carlo, M. Sergi, D. Compagnone, A. Escarpa, Press-transferred carbon black nanoparticles on board of microfluidic chips for rapid and sensitive amperometric determination of phenyl carbamate pesticides in environmental samples, Microchim. Acta. 183 (2016) 3143-3149. doi:10.1007/s00604016-1964-7. 
[43] M. Pumera, A.H. Loo, Layered transition-metal dichalcogenides (MoS2and WS2) for sensing and biosensing, TrAC - Trends Anal. Chem. 61 (2014) 49-53. doi:10.1016/j.trac.2014.05.009.

[44] M.A. Lukowski, A.S. Daniel, F. Meng, A. Forticaux, L. Li, S. Jin, Enhanced hydrogen evolution catalysis from chemically exfoliated metallic MoS2 nanosheets, J. Am. Chem. Soc. 135 (2013) 10274-10277. doi:10.1021/ja404523s.

[45] Y. Lin, X. Chen, Y. Lin, Q. Zhou, D. Tang, Non-enzymatic sensing of hydrogen peroxide using a glassy carbon electrode modified with a nanocomposite made from carbon nanotubes and molybdenum disulfide, Microchim. Acta. 182 (2015) 1803-1809. doi:10.1007/s00604-015-1517-5.

[46] N. Lingappan, N.H. Van, S. Lee, D.J. Kang, Growth of three dimensional flower-like molybdenum disulfide hierarchical structures on graphene/carbon nanotube network: An advanced heterostructure for energy storage devices, J. Power Sources. 280 (2015) 39-46. doi:10.1016/j.jpowsour.2015.01.064.

[47] I. Song, C. Park, H.C. Choi, W. Zhang, P. Zhang, Z. Su, G. Wei, N.A. Kumar, M.A. Dar, R. Gul, J.B. Baek, M. Pumera, A.H. Loo, K.J. Huang, L.L.L. Wang, J.Z. Zhang, L.L.L. Wang, Y.P. Mo, G.W. Shi, Y.M. Liu, A.M. Van Der Zande, P.Y. Huang, D.A. Chenet, T.C. Berkelbach, Y. You, G.H. Lee, T.F. Heinz, D.R. Reichman, D.A. Muller, J.C. Hone, K. Lee, R. Gatensby, N. McEvoy, T. Hallam, G.S. Duesberg, K.G. Zhou, N.N. Mao, H.X. Wang, Y. Peng, H.L. Zhang, K. Lee, H.Y. Kim, M. Lotya, J.N. Coleman, G.T. Kim, G.S. Duesberg, Hydrothermal synthesis of molybdenum disulfide nanosheets as supercapacitors electrode material, Adv. Mater. 50 (2014) 554-561. doi:10.1002/adma.201101013.

[48] K.J. Huang, L. Wang, J.Z. Zhang, L.L. Wang, Y.P. Mo, One-step preparation of layered molybdenum disulfide/multi-walled carbon nanotube composites for enhanced performance supercapacitor, Energy. 67 (2014) 234-240. doi:10.3354/dao02982.

[49] W. Zhang, P. Zhang, Z. Su, G. Wei, Synthesis and sensor applications of $\mathrm{MoS}_{2}$-based nanocomposites, Nanoscale. 7 (2015) 18364-18378. doi:10.1039/C5NR06121K.

[50] K.J. Huang, L. Wang, J. Li, Y.M. Liu, Electrochemical sensing based on layered MoS2-graphene composites, Sensors Actuators, B Chem. 178 (2013) 671-677. doi:10.1016/j.snb.2013.01.028.

[51] A. Sinha, Dhanjai, B. Tan, Y. Huang, H. Zhao, X. Dang, J. Chen, R. Jain, MoS2 nanostructures for electrochemical sensing of multidisciplinary targets: A review, TrAC - Trends Anal. Chem. 102 (2018) 75-90. doi:10.1016/j.trac.2018.01.008.

[52] D. Talarico, F. Arduini, A. Constantino, M. Del Carlo, D. Compagnone, D. Moscone, G. Palleschi, Carbon black as successful screen-printed electrode modifier for phenolic compound detection, Electrochem. Commun. 16 (2015) 28-32. doi:10.1016/j.elecom.2015.08.010.

[53] F. Della Pelle, R. Di Battista, L. Vázquez, F.J. Palomares, M. Del Carlo, M. Sergi, D. Compagnone, A. Escarpa, Press-transferred carbon black nanoparticles for class-selective antioxidant electrochemical detection, Appl. Mater. Today. 9 (2017) 29-36. doi:10.1016/j.apmt.2017.04.012.

[54] I. Vasilescu, S.A.V. Eremia, M. Kusko, A. Radoi, E. Vasile, G.-L. Radu, Molybdenum disulphide and graphene quantum dots as electrode modifiers for laccase biosensor, Biosens. Bioelectron. 75 (2016) 232-237. doi:10.1016/J.BIOS.2015.08.051.

[55] H. Huang, J. Zhang, M. Cheng, K. Liu, X. Wang, Amperometric sensing of hydroquinone using a glassy carbon electrode modified with a composite consisting of graphene and molybdenum disulfide, Microchim. Acta. 184 (2017) 4803-4808. doi:10.1007/s00604-017-2531-6.

[56] S. Ji, Z. Yang, C. Zhang, Y.E. Miao, W.W. Tjiu, J. Pan, T. Liu, Nonenzymatic sensor for glucose based on a glassy carbon electrode modified with $\mathrm{Ni}(\mathrm{OH}) 2$ nanoparticles grown on a film of molybdenum sulfide, Microchim. Acta. 180 (2013) 1127-1134. doi:10.1007/s00604-013-1035-2.

[57] V. Mani, M. Govindasamy, S.-M.M. Chen, R. Karthik, S.-T.T. Huang, Determination of dopamine using a glassy carbon electrode modified with a graphene and carbon nanotube hybrid decorated with molybdenum disulfide flowers, Microchim. Acta. 183 (2016) 2267-2275. doi:10.1007/s00604-016-1864-x.

[58] X. Li, X. Du, Molybdenum disulfide nanosheets supported Au-Pd bimetallic nanoparticles for non-enzymatic electrochemical sensing of hydrogen peroxide and glucose, Sensors Actuators, B Chem. 239 (2017) 536-543. doi:10.1016/j.snb.2016.08.048.

[59] L. Gu, S.E. House, X. Wu, B. Ou, R.L. Prior, Procyanidin and catechin contents and antioxidant capacity of cocoa and chocolate products, J. Agric. Food Chem. 54 (2006) 4057-4061. doi:10.1021/jf060360r.

[60] C.A. Schneider, W.S. Rasband, K.W. Eliceiri, NIH Image to ImageJ: 25 years of image analysis., Nat. Methods. 9 (2012) 671-675.

[61] R. Re, N. Pellegrini, A. Proteggente, A. Pannala, M. Yang, C. Rice-Evans, Antioxidant activity applying an improved ABTS radical cation decolorization assay, Free Radic. Biol. Med. 26 (1999) 1231-1237. doi:10.1016/S0891-5849(98)00315-3.

[62] M. Del Carlo, A. Amine, M. Haddam, F. Della Pelle, G.C. Fusella, D. Compagnone, Selective Voltammetric Analysis of o-Diphenols from Olive Oil Using Na2MoO4 as Electrochemical Mediator, Electroanalysis. 24 (2012). doi:10.1002/elan.201100603.

[63] F. Della Pelle, A. Scroccarello, M. Sergi, M. Mascini, M. Del Carlo, D. Compagnone, Simple and rapid silver nanoparticles based antioxidant capacity assays: Reactivity study for phenolic compounds, Food Chem. 256 (2018) 342-349. doi:10.1016/j.foodchem.2018.02.141. 
[64] F. Della Pelle, D. Vilela, M.C. Gonzàlez, C. Lo Sterzo, D. Compagnone, M. Del Carlo, A. Escarpa, Antioxidant capacity index based on gold nanoparticles formation. Application to extra virgin olive oil samples, Food Chem. 178 (2015) 70-75. doi:10.1016/j.foodchem.2015.01.045.

[65] A. Scroccarello, F. Della Pelle, L.N. Neri, P. Pittia, D. Compagnone, Silver and gold nanoparticles based colorimetric assays for the determination of sugars and polyphenols in apples, Food Res. Int. 119 (2019) 359368. doi:https://doi.org/10.1016/j.foodres.2019.02.006.

[66] L. Blandón-Naranjo, J. Hoyos-Arbeláez, M. V. Vázquez, F. Della Pelle, D. Compagnone, NADH Oxidation onto different carbon-based sensors: Effect of structure and surface-oxygenated groups, J. Sensors. 2018 (2018). doi:10.1155/2018/6525919.

[67] F. Della Pelle, C. Angelini, M. Sergi, M. Del Carlo, A. Pepe, D. Compagnone, Nano carbon black-based screen printed sensor for carbofuran, isoprocarb, carbaryl and fenobucarb detection: application to grain samples, Talanta. 186 (2018) 389-396. doi:10.1016/j.talanta.2018.04.082.

[68] F. Della Pelle, L. Vázquez, M. Del Carlo, M. Sergi, D. Compagnone, A. Escarpa, Press-Printed Conductive Carbon Black Nanoparticle Films for Molecular Detection at the Microscale, Chem. - A Eur. J. 22 (2016) 12761-12766. doi:10.1002/chem.201601743.

[69] Y. Li, Y. Feng, S. Zhu, C. Luo, J. Ma, F. Zhong, Journal of Food Composition and Analysis The effect of alkalization on the bioactive and flavor related components in commercial cocoa powder, J. Food Compos. Anal. 25 (2012) 17-23. doi:10.1016/j.jfca.2011.04.010.

[70] I. Novak, M. Šeruga, Š. Komorsky-Lovrić, Square-wave and cyclic voltammetry of epicatechin gallate on glassy carbon electrode, J. Electroanal. Chem. 631 (2009) 71-75. doi:10.1016/j.jelechem.2009.03.005. 


\section{Figure Captions}

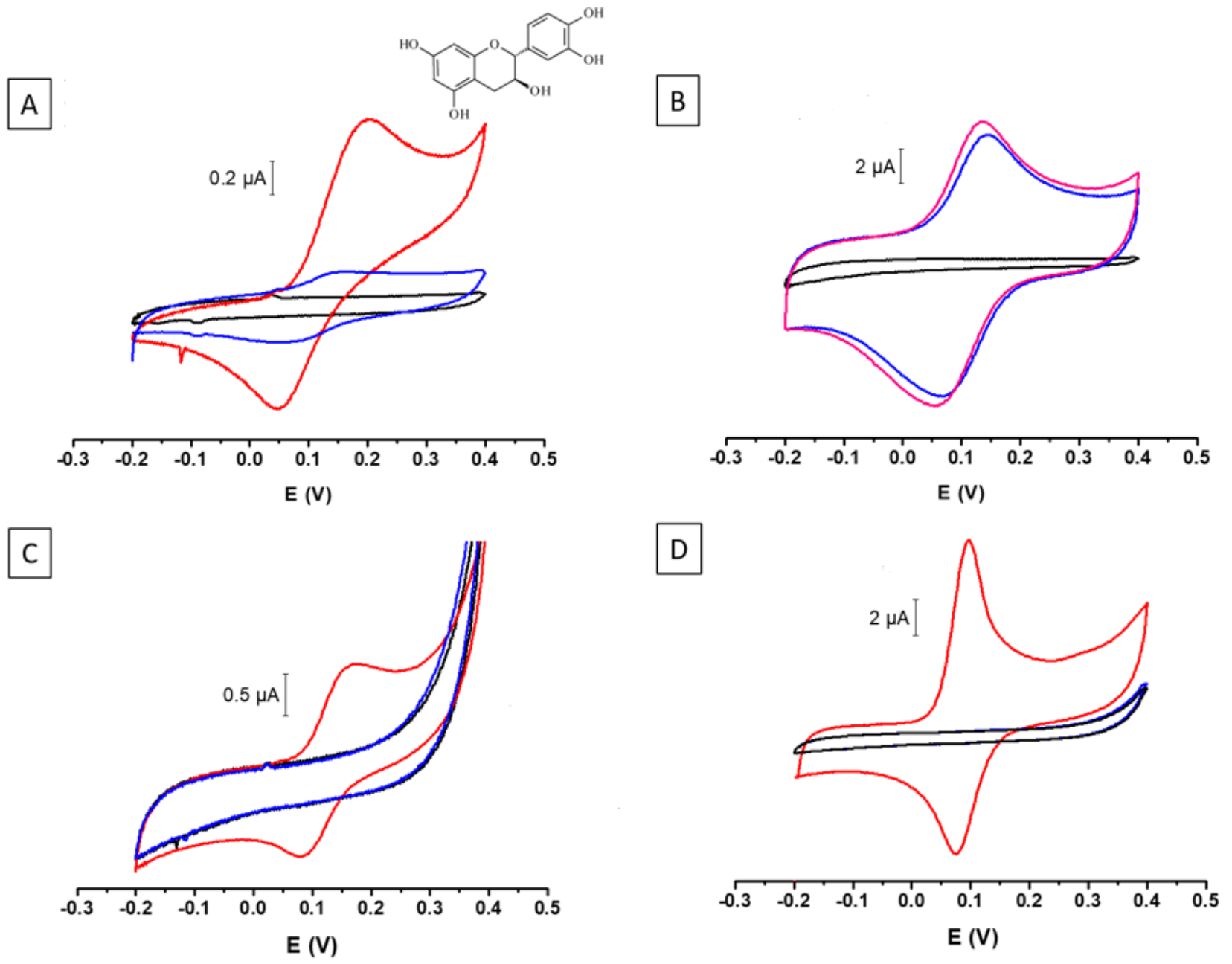

Fig. 1. Cyclic voltammograms of the bare SPE (A), SPE-CB (B), SPE-MoS $2(\mathbf{C})$, and SPE-CB/MoS $(\mathbf{D})$. Black line CV in $0.1 \mathrm{mmol} \mathrm{L}^{-1} \mathrm{~PB}(\mathrm{pH} 7)+0.1 \mathrm{~mol} \mathrm{~L}^{-1} \mathrm{KCl}$, before catechin $\mathrm{CV}$. Red line $\mathrm{CV}$ of $50 \mu \mathrm{mol} \mathrm{L}^{-1}$ catechins (prepared in 0.1 mmol L ${ }^{-1} \mathrm{~PB}+0.1 \mathrm{~mol} \mathrm{~L}^{-1} \mathrm{KCl}, \mathrm{pH}$ 7). Blue line $\mathrm{CV}$ in $0.1 \mathrm{mmol} \mathrm{L}^{-1} \mathrm{~PB}, \mathrm{pH} 7+0.1 \mathrm{~mol} \mathrm{~L}^{-1} \mathrm{KCl}$, after catechins $\mathrm{CV}$. CVs performed at a scan rate of $50 \mathrm{mV} \mathrm{s}^{-1}$.

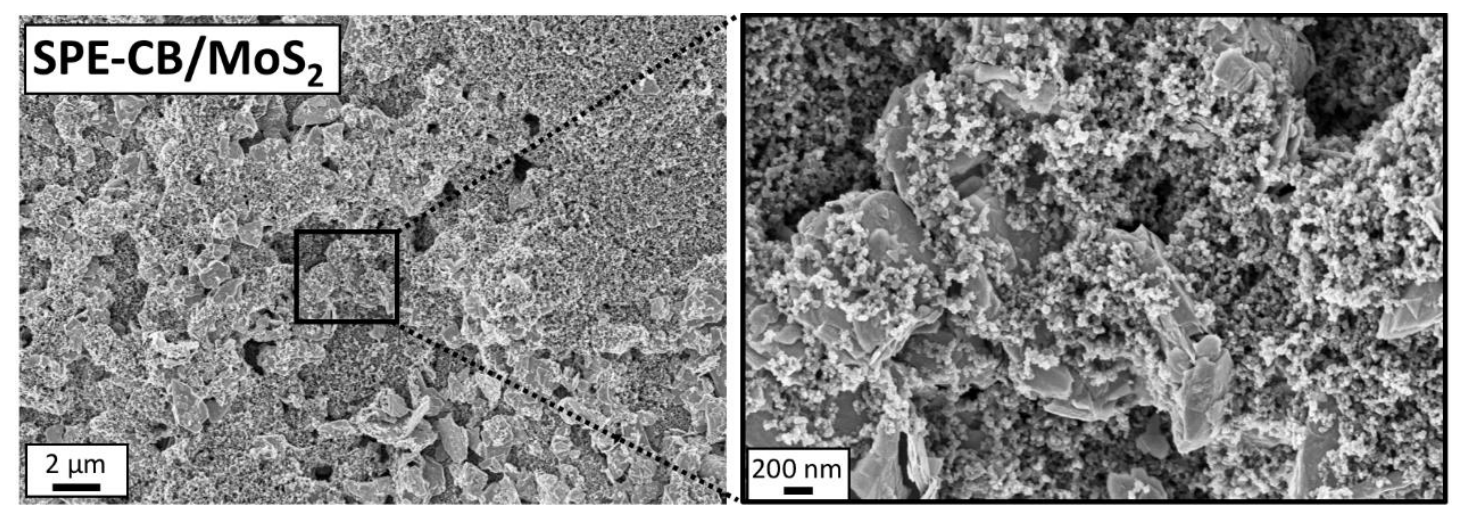

Fig. 2. SEM micrograph and magnification of the SPE-CB/MoS 2 . 

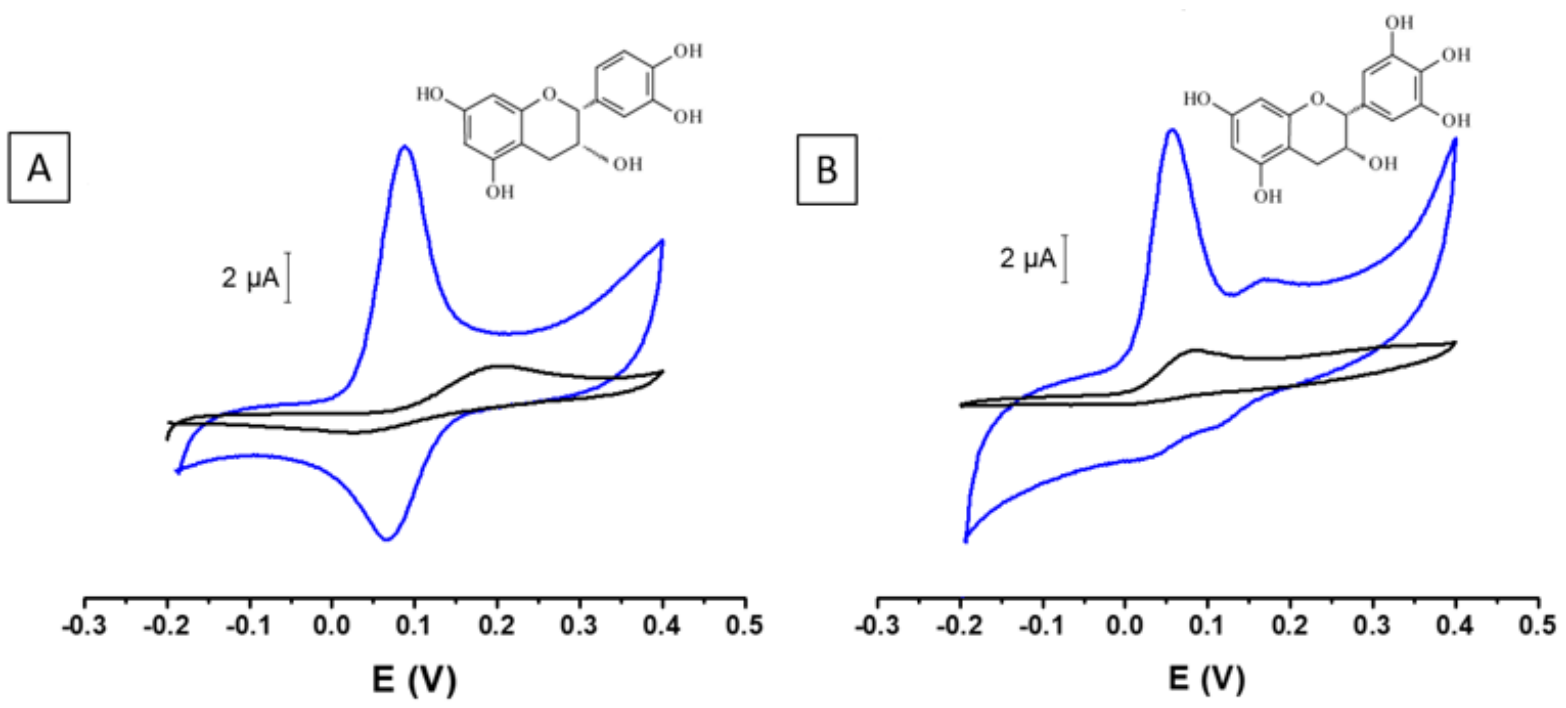

Fig. 3. Cyclic voltammograms of the bare SPE (black line) and SPE-CB/MoS (blue line) performed with $50 \mu \mathrm{mol} \mathrm{L}^{-1}$ epicatechin (A) and epigallocatechin $(\mathbf{B})$ prepared in $0.1 \mathrm{mmol} \mathrm{L}^{-1} \mathrm{~PB}+0.1 \mathrm{~mol} \mathrm{~L}^{-1} \mathrm{KCl}(\mathrm{pH} 7)$ at a scan rate of $50 \mathrm{mV}$ $\mathrm{s}^{-1}$.
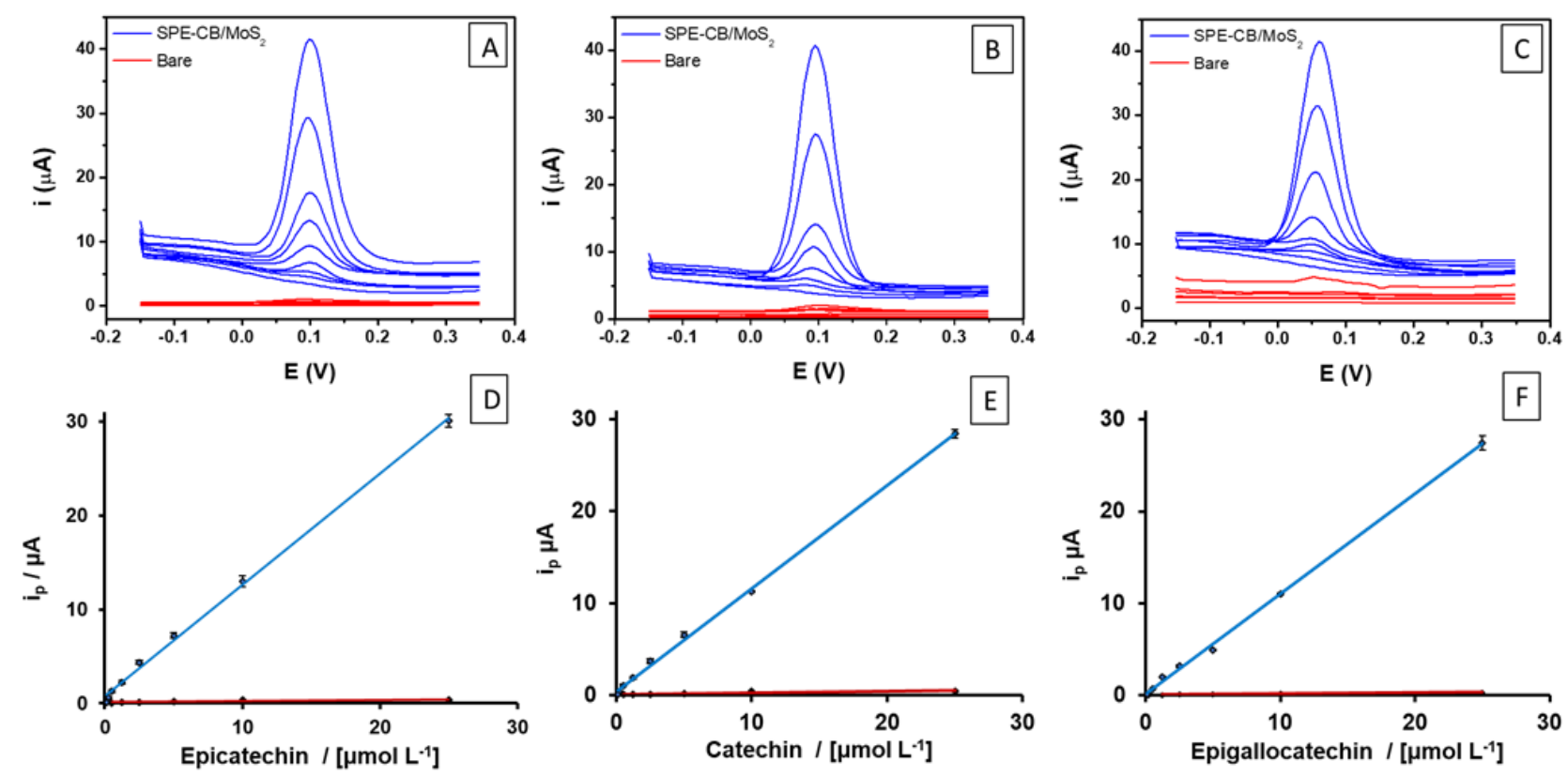

Fig. 4. (A-C) DPV curves obtained using bare SPE (red line) and CB-SPE/MoS (blue line) in presence of increasing concentrations of epicatechin (A), catechin $(\mathbf{B})$ and epigallocatechin $(\mathbf{C})$. (D-F) DPV calibration curves (mean value of three repetitions) obtained using bare SPE (red line) and CB-SPE/MoS ${ }_{2}$ (blue line) in presence of increasing concentrations of epicatechin $(\mathbf{D})$, catechin $(\mathbf{E})$ and epigallocatechin $(\mathbf{F})$. For each analyte, the calibration was performed in triplicate, the resulting linear equation (obtained with the mean values, $n=3$ ) and determination coefficient obtained for the SPE-CB/MoS 2 are reported in Table 1; while for the bare SPE: $(C T) y=0.0168 x+0.0798, R^{2}=0.7382 ;(E P) y=$ $0.0129 \mathrm{x}+0.1256, \mathrm{R}^{2}=0.7316 ;(\mathrm{EG}) \mathrm{y}=0.0107 \mathrm{x}+0.048, \mathrm{R}^{2}=0.9406$. (A-F) DPV conditions: pulse width $50 \mathrm{~ms}$, pulse amplitude $20 \mathrm{mV}$. The standards were prepared in phosphate-buffered $0.01 \mathrm{mmol} \mathrm{L}^{-1} \mathrm{~PB}+0.1 \mathrm{~mol} \mathrm{~L}^{-1} \mathrm{KCl}(\mathrm{pH} 7)$. 
A

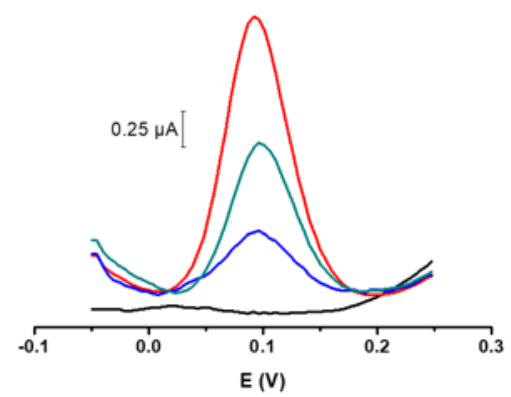

B

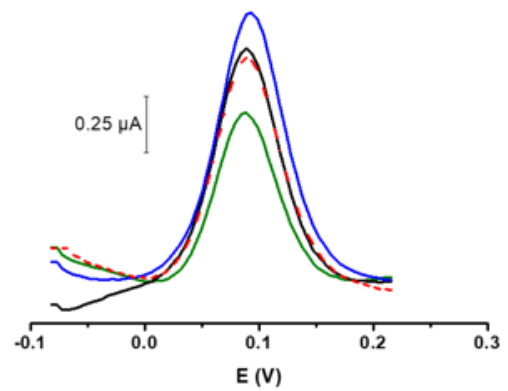

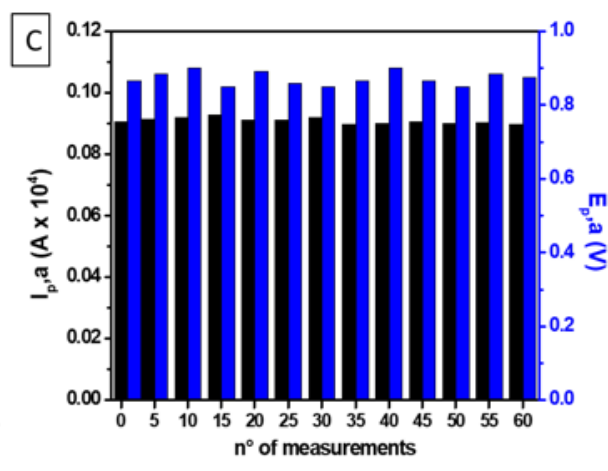

Fig. 5. (A) DPV obtained analyzing a mix of cocoa samples (blue line), fortified with $0.25 \mu \mathrm{mol} \mathrm{L}^{-1}$ (green line) and 0.5 $\mu \mathrm{mol} \mathrm{L}{ }^{-1}$ (red line) of epicatechin. (B) DPV of three different samples with low (sample 12, green line), medium (sample 5 , black line) and high (sample 1, blue line) polyphenols content. The dashed red line represents the sample 5 measurement repetition, performed after the measurement of the whole set of samples $\left(n^{\circ}\right.$ 59). (C) DPV Oxidation currents and oxidation potentials obtained with $10 \mathrm{uM}$ epicatechin, used to monitor the $\mathrm{SPE}-\mathrm{CB} / \mathrm{MoS}_{2}$ response, during the samples analyzed. Each 5 samples analyzed the measurement has been performed. 


\section{Supplementary material}

\section{High-performance carbon black/molybdenum disulfide nanohybrid sensor for cocoa catechins determination using an extraction-free approach}

Flavio Della Pelle ${ }^{\mathrm{a}}$, Daniel Rojas ${ }^{\mathrm{a}, \mathrm{b}}$, Annalisa Scroccarello ${ }^{\mathrm{a}}$, Michele Del Carlo ${ }^{\mathrm{a}}$, Giovanni Ferraro ${ }^{\mathrm{c}}$, Carla Di Mattia ${ }^{\mathrm{a}}$, Maria Martuscelli ${ }^{\mathrm{a}}$, Alberto Escarpa ${ }^{\mathrm{b}, \mathrm{d}^{*}}$, Dario Compagnone $\mathrm{a}^{\mathrm{a}^{* *}}$

${ }^{\text {a }}$ Faculty of Bioscience and Technology for Food, Agriculture and Environment University of Teramo 64023, Teramo (Italy). Fax:(+39) 0861-266942, e-mail:**dcompagnone@unite.it

${ }^{\mathrm{b}}$ Department of Analytical Chemistry, Physical Chemistry and Chemical Engineering, Faculty of Sciences University of Alcalá, E-28871 Alcalá de Henares, Madrid, Spain. Fax: (+34) 918854971, e-mail: *alberto.escarpa@uah.es

${ }^{c}$ Department of Chemistry “Ugo Schiff” and CSGI, University of Florence, Via della Lastruccia 3-Sesto Fiorentino, I50019, Florence, Italy.

dChemical Research Institute “Andres M. del Rio”, University of Alcalá, E-28871, Madrid, Spain

\section{$\underline{\text { S1 Catechin electrochemical behavior on a bare screen-printed electrode }}$}

In order to study the catechin (CT) electrochemical behavior 4 consecutive CVs have been carried out using a bare SPE and after each scan an EIS spectra has been recorded (Fig. S1). The first cyclic voltammogram of CT performed using a bare SPE (Fig. S1A, blue line), shows two anodic peaks, the potentials of the first (peak 1) and the second peak (peak 2) are $0.188 \mathrm{~V}$ and $0.481 \mathrm{~V}$, respectively. A cathodic peak (peak 3 ) is observed at $-0.015 \mathrm{~V}$; this reduction peak is related to the first oxidation peak which is a reversible reaction [1]. According to the literature, the oxidation mechanism proceeds in sequential steps, related with the catechol moiety (peak 1 oxidation and peak 3 reduction) and resorcinol group (peak 2) [1]. The first peak (catechol moiety) is higher compared with the current of resorcinol (peak 2) and remains in following scans; both behaviors are in agreement with the higher radical scavenging activity corresponding to ortho-diphenolic structures [1-5]. Noteworthy, in the following scans, the passivation process results evident, the oxidation product is not electroactive and passives more and more the electrode surface. This behavior is already reported by Brett group [1] onto a glassy carbon electrode. In fact, in this study is proved that the CT resorcinol group is irreversibly oxidized and the catechin moiety results strongly adsorbed on the electrode surface. The CT passivation [1,6,7] was also confirmed by EIS using $\mathrm{Fe}(\mathrm{CN})_{6}{ }^{4-13-}$. Nyquist plots (Fig. S1B) obtained by EIS exhibited a progressive increase of resistance to charge transfer $\left(\mathrm{R}_{\mathrm{ct}}\right)$ scan by scan, in accordance with the CVs of CT (Fig. S1A). $\mathrm{R}_{\mathrm{ct}}$ increased (Fig. 1C) from $1.1 \times 10^{3} \Omega($ before the first scan) to $4.1 \times 10^{4} \Omega$ (after 4 scans), clearly indicating a passivation process. Definitely, the CT strongly adsorbed on the SPE electrode surface and the final product is not electroactive and blocks the electrode surface.
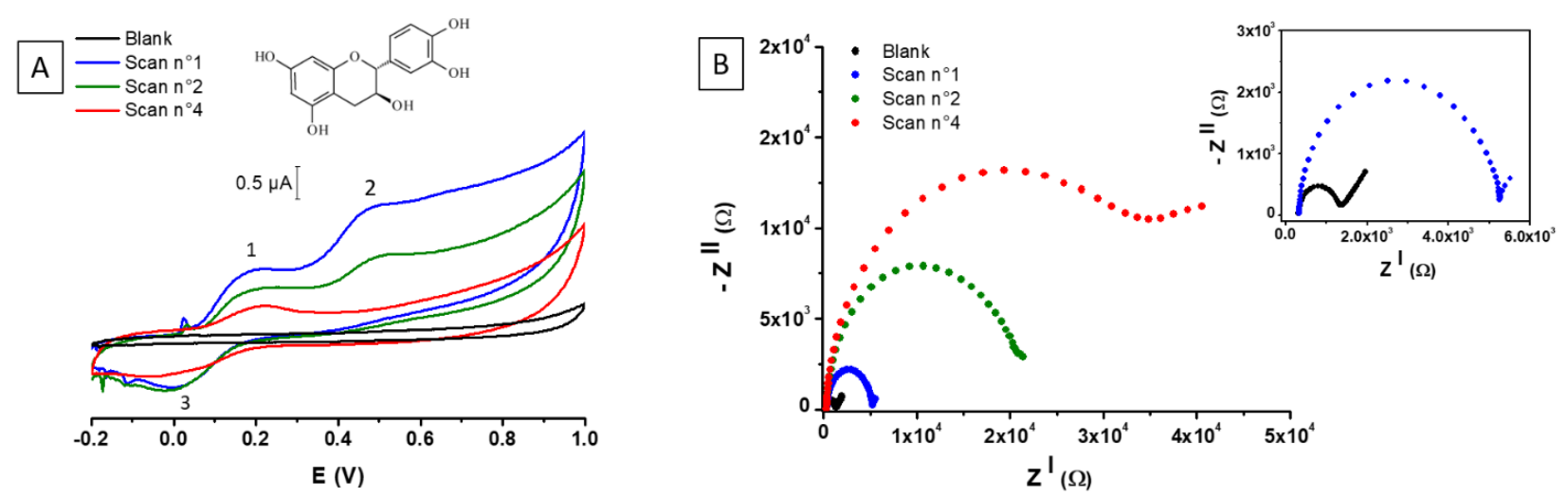
Fig. S1. (A) CVs of catechin (blue line) at $50 \mu \mathrm{mol} \mathrm{L}{ }^{-1}$ performed with a bare SPE: 1st scan (blue line), 2nd scan (green line), 4th scan (red line), in black the scan performed before the catechin $\mathrm{CV}$. CV performed in phosphate buffer $10 \mathrm{mmol} \mathrm{L}^{-1}+0.1 \mathrm{~mol} \mathrm{~L}^{-1} \mathrm{KCl}_{\text {at }} \mathrm{pH} 7.0 \mathrm{with}$ a scan rate of 50 $\mathrm{mV} \mathrm{s}^{-1}$. (B) Nyquist plots of $5 \mathrm{mmol} \mathrm{L}{ }^{-1} \mathrm{Fe}(\mathrm{CN})_{6}^{4-/ 3-}$ in $0.1 \mathrm{KCl} \mathrm{mol} \mathrm{L}{ }^{-1}$ performed obtained with a bare SPE after the catechin $\mathrm{CVs}$ analysis reported in Figure 1A. After the1st scan (blue circles), 2nd scan (green circles), 4th scan (red circles), in black the Nyquist plot obtained before the catechin CV. In the inset a magnification of the Nyquist obtained before the CT CV (blank line) and after the $1^{\circ}$ st scan (blue line)

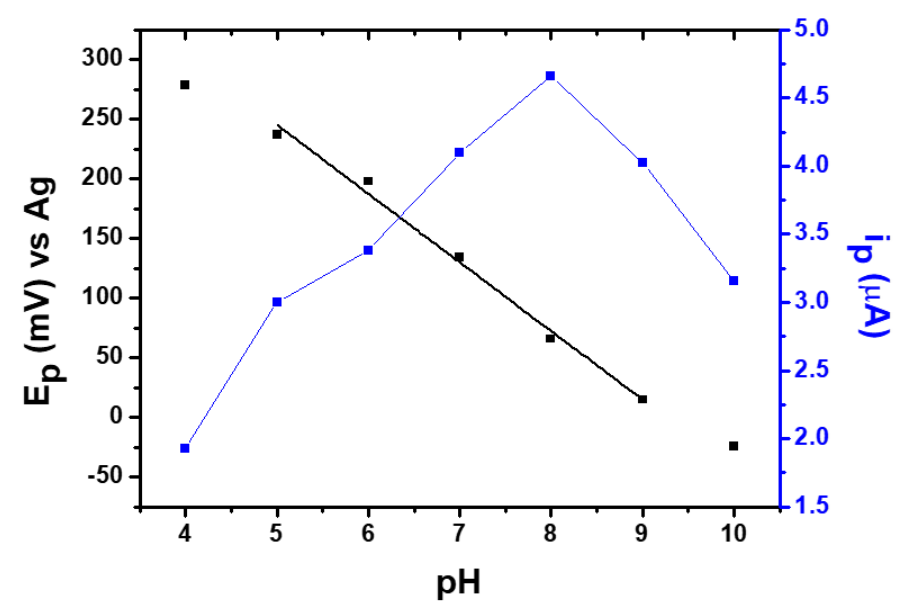

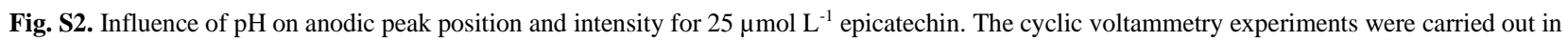
phosphate buffer at a scan rate of $25 \mathrm{mV} / \mathrm{s}$.

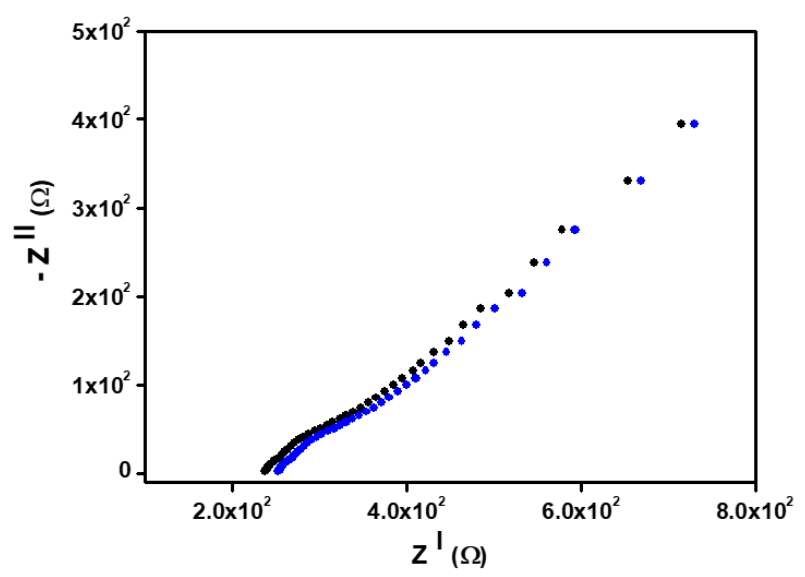

Fig. S3. Nyquist plots of $5 \mathrm{mmol} \mathrm{L}^{-1} \mathrm{Fe}(\mathrm{CN})_{6}{ }^{4-/ 3-}$ in $0.1 \mathrm{KCl} \mathrm{mol} \mathrm{L}^{-1}$ obtained after (black circles) and before (blue circles) 4 consecutive $50 \mu \mathrm{mol} \mathrm{L}{ }^{-1}$ catechin cyclic voltammetry scans, using the SPE-CB/MoS 2 .

\section{S2 Morphological analysis}



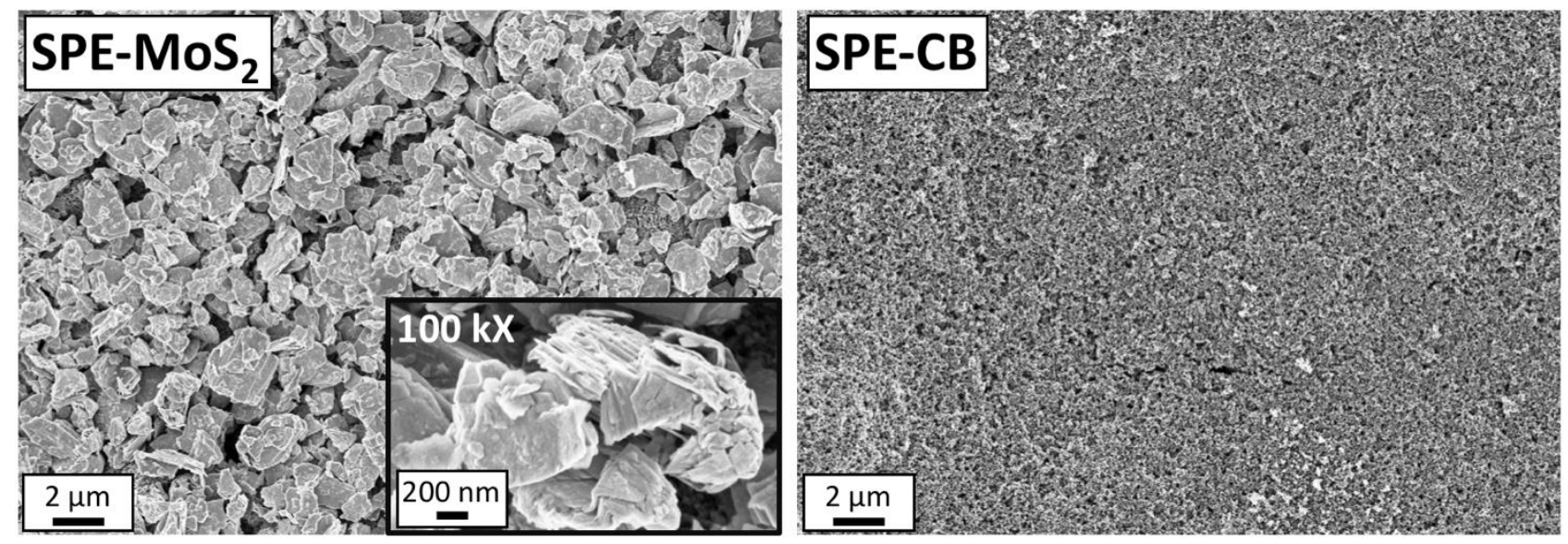

Fig. S4. SEM micrographs of the SPE-MoS 2 and SPE-CB.
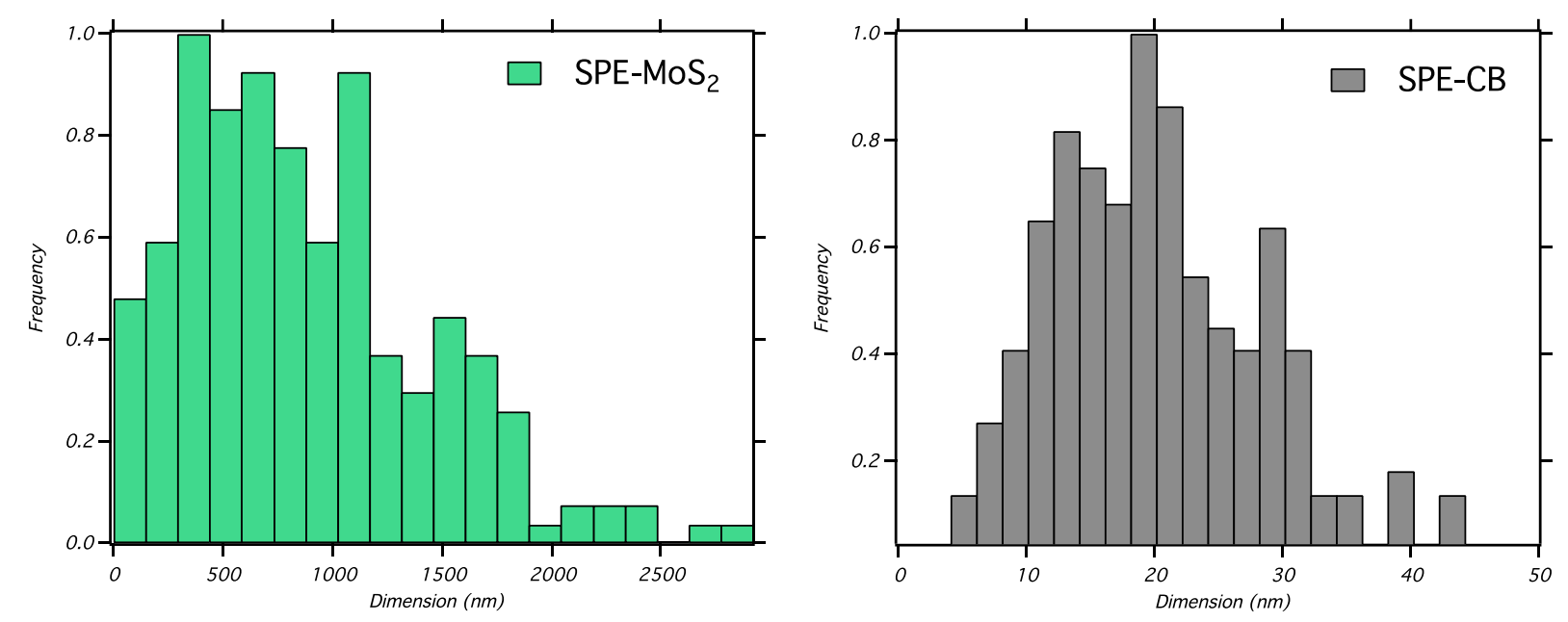

Fig. S5 Particle size distributions obtained from the image analysis for the samples SPE-MoS $\mathrm{S}_{2}$ and SPE-CB. The distributions are calculated by measuring a consistent number of particles (more than 200 particles in the case of the sample SPE-MoS 2 and more than 400 for the SPE-CB sample).

Surface homogeneity can be obtained by using the lacunarity descriptor [8], which we calculated using the FracLac tool package [9] contained in ImageJ. The image lacunarity, $\Lambda$, is defined as the gappiness or the heterogeneity associated to an image: low values of lacunarity correspond to homogeneous systems.

Lacunarity can be calculated as follows:

$$
\Lambda=\frac{\sum \lambda_{g}}{N_{g}}
$$

where $\mathrm{N}_{\mathrm{g}}$ is the number of orientations of the grid and $\lambda_{g}$ is defined as:

$$
\lambda_{\varepsilon, g}=\left(\frac{\sigma}{\mu}\right)_{\varepsilon, g}^{2}
$$

$\varepsilon$ is the box dimension, $\mathrm{g}$ is the number of all possible orientations of the grid constituted by the different boxes, $\sigma$ is the standard deviation of pixels per box and $\mu$ is the mean density of black pixels per box. Thus, there is a $\lambda$ value for each $\varepsilon$ in each series of grid size and for each g, in a set of grid orientations. 
In our case, the lacunarity of the image was calculated by using the "Box Counting" algorithm with an $\varepsilon$ value within 4 of the pixel size and a number of orientations, $\mathrm{N}_{\mathrm{g}}$, equal to 9 . These parameters were adjusted in order to measure nanometric inhomogeneities. The calculation was performed on the micrographs with a magnification of $10 \mathrm{KX}$.
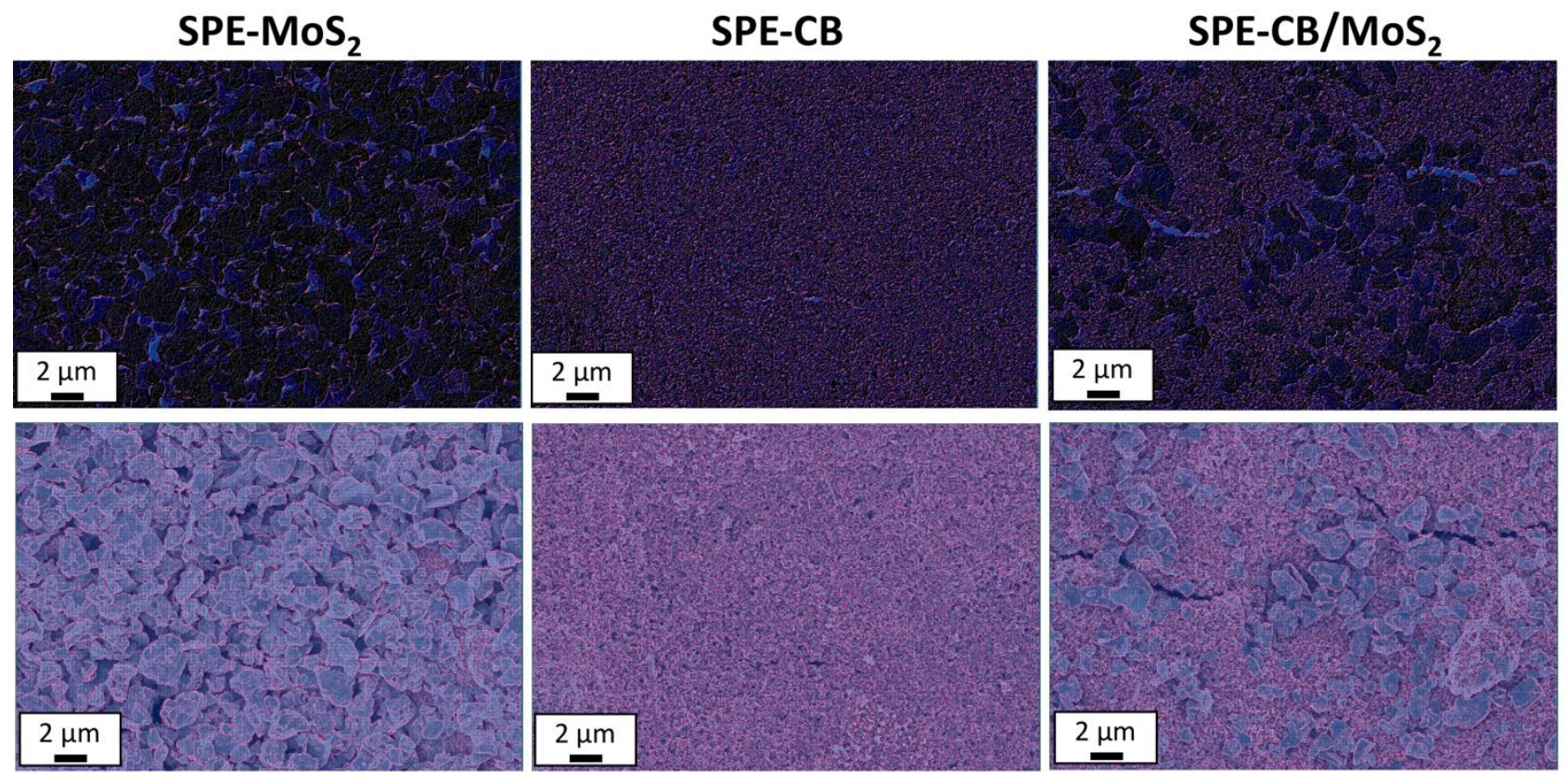

Fig. S6. Top: Lacunarity grids obtained from the analysis of the SEM micrographs acquired on the different electrodes. Red corresponds to low values of lacunarity, blue indicates high lacunarity. Bottom: Overlay of lacunarity grids and SEM micrographs of all the investigated samples.

The lacunarity values obtained for the different samples are $0.57,0.23$ and 0.37 for SPE-MoS 2 SPE-CB and SPE$\mathrm{CB} / \mathrm{MoS}_{2}$, respectively.

A

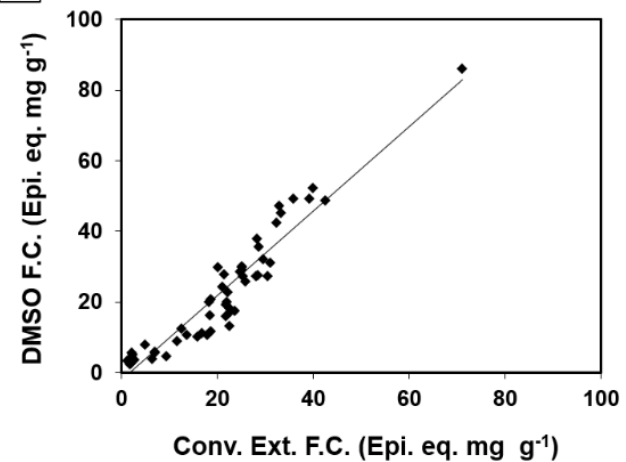

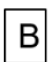

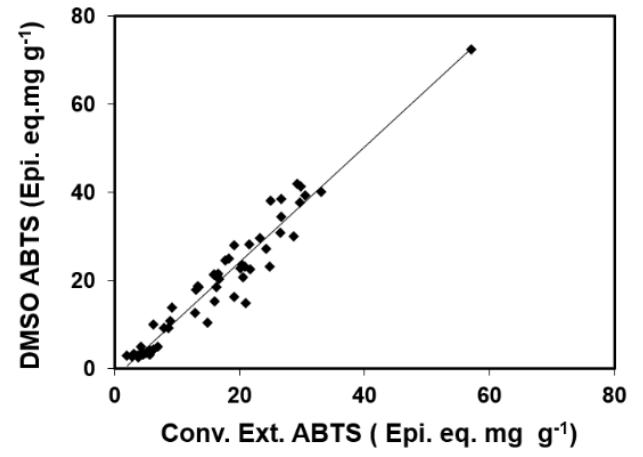

Fig. S7. Correlation curves between the data obtained (mean values, $n=3$ ), analyzing the 59 cocoa powder samples, extract with the proposed method based on DMSO and the conventional extraction (Conc.Ext.) method. Extracts analyzed using the Folin-Ciocalteu method (A) and ABTS assay (B). All the data are expressed in epicatechin equivalents.

\section{References}

[1] P. Janeiro, A.M. Oliveira Brett, Catechin electrochemical oxidation mechanisms, Anal. Chim. Acta. 518 (2004) 109-115. doi:10.1016/j.aca.2004.05.038. 
[2] M. Del Carlo, A. Amine, M. Haddam, F. Della Pelle, G.C. Fusella, D. Compagnone, Selective Voltammetric Analysis of o-Diphenols from Olive Oil Using Na2MoO4 as Electrochemical Mediator, Electroanalysis. 24 (2012). doi:10.1002/elan.201100603.

[3] F. Della Pelle, R. Di Battista, L. Vázquez, F.J. Palomares, M. Del Carlo, M. Sergi, D. Compagnone, A. Escarpa, Press-transferred carbon black nanoparticles for class-selective antioxidant electrochemical detection, Appl. Mater. Today. 9 (2017) 29-36. doi:10.1016/j.apmt.2017.04.012.

[4] F. Della Pelle, A. Scroccarello, M. Sergi, M. Mascini, M. Del Carlo, D. Compagnone, Simple and rapid silver nanoparticles based antioxidant capacity assays: Reactivity study for phenolic compounds, Food Chem. 256 (2018) 342-349. doi:10.1016/j.foodchem.2018.02.141.

[5] F. Della Pelle, D. Vilela, M.C. Gonzàlez, C. Lo Sterzo, D. Compagnone, M. Del Carlo, A. Escarpa, Antioxidant capacity index based on gold nanoparticles formation. Application to extra virgin olive oil samples, Food Chem. 178 (2015) 70-75. doi:10.1016/j.foodchem.2015.01.045.

[6] F. Della Pelle, D. Compagnone, Nanomaterial-based sensing and biosensing of phenolic compounds and related antioxidant capacity in food, Sensors. 18 (2018) 462. doi:10.3390/s18020462.

[7] T.A. Enache, A.M. Oliveira-brett, Phenol and para -substituted phenols electrochemical oxidation pathways, J. Electroanal. Chem. 655 (2011) 9-16. doi:10.1016/j.jelechem.2011.02.022.

[8] M.N. Barros Filho, F.J. Sobreira, Accuracy of lacunarity algorithms in Texture Classification of high spatial resolution images from urban areas, Int. Arch. Photogramm. Remote Senising Spat. Inf. Sci. 37 (2008) 417422.

[9] A.L. Karperien, FracLac for ImageJ, Loop. (2013). 